\title{
An efficient genetic algorithm for large-scale planning of dense and robust industrial wireless networks
}

\author{
Xu Gong a,*, David Plets ${ }^{a}$, Emmeric Tanghe ${ }^{\text {a }}$, Toon De Pessemier ${ }^{\text {a }}$, Luc Martens ${ }^{\text {a }}$, \\ Wout Joseph ${ }^{\text {a }}$ \\ ${ }^{a}$ Department of Information Technology, Ghent University/imec, Technologiepark 15, 9052 Ghent, Belgium \\ * E-mail: xu.gong@ugent.be. Tel.: +32 09264 3325. Fax.: +32 93314899. \\ E-mail of co-authors: david.plets@ugent.be,emmeric.tanghe@ugent.be, toon.depessemier@ugent.be, \\ luc.martens@intec.ugent.be,wout.joseph@ugent.be
}

\begin{abstract}
With the penetration of Internet of things in manufacturing industry, it is an unavoidable issue to maintain robust wireless connections among machines and human workers in harsh industrial environments. However, the existing wireless planning tools focus on office environments without considering shadowing effects of prevalent metal obstacles. To fill this gap, this paper proposes an over-dimensioning (OD) model, which automates the decision making on deploying a robust industrial wireless local area network (IWLAN). This model creates two full coverage layers while minimizing the deployment cost, and guaranteeing a minimal separation distance between two access points (APs) to prevent APs that cover the same region from being simultaneously shadowed by an obstacle. Moreover, an empirical one-slope path loss model, which considers three-dimensional obstacle shadowing effects, is proposed for simple yet precise coverage calculation. To solve this OD model even at a large size, an efficient genetic algorithm based over-dimensioning (GAOD) algorithm is designed. Genetic operators, parallelism, and speedup measures are tailored to enable large-scale optimization. A greedy heuristic based over-dimensioning (GHOD) algorithm is further proposed, as a state-of-the-art heuristic benchmark algorithm. In small- and large-size OD problems based on industrial data, the GAOD was demonstrated to be $20 \%-25 \%$ more economical than benchmark algorithms for OD in the same environment. The effectiveness of GAOD was further experimentally validated with a real deployment system. Though this paper focuses on an IWLAN, the proposed GAOD can serve as a decision making tool for deploying robust industrial wireless networks of many other types, such as wireless sensor networks and RFID networks.
\end{abstract}

Keywords: Genetic algorithms, large-scale optimization, wireless network deployment, Internet of things, cyber physical systems 


\section{Introduction}

Wireless technologies are penetrating to factories for industrial Internet of things, due to the advantages that they possess over cabled technologies, such as mobility, flexibility, coverage over hard-to-reach locations, as well as lower installation and maintenance cost. For instance, a WiFi network on the shop floor connects devices, such as handheld RFID readers, power meters, wireless sensors, and laptops, to the industrial management systems. This further facilitates onsite operators and factory managers to reconfigure the control software and change the operating parameters (Cena et al., 2010). Furthermore, wireless technologies play as an indispensable role in factories of the future, such as Industry 4.0 and Industrial Internet (Gong et al., 2016b).

To deploy a wireless network in a target environment, a human network planner may perform a site survey to empirically determine the number and location of wireless nodes (Plets et al., 2016). Evidently, this is rather a trialand-error approach, which is inefficient. Fortunately, a wireless network planning tool automates and enhances this decision making, by recommending an optimal or nearly-optimal deployment solution for a target environment. In this way, a human network planner only needs to place each wireless node according to this obtained solution. Both the deployment time and the deployment cost are thereby reduced. The contribution of such an automated wireless network planning tool is even strengthened when applying to an industrial indoor environment, which can be super large and otherwise requires extensive manual site survey work.

Nevertheless, an industrial indoor environment is harsh for radio propagation (Gong et al., 2016b; Tanghe et al., 2014), compared to office environments on which most wireless network planning tools focus. It is dominated by various metal or steel objects, such as production machines, storage racks, materials (steel bars, metal plates, etc.), and vehicles (automated guided vehicles or AGVs, cranes and forklifts). These obstacles shadow the radio propagation and cause coverage holes on desired areas. According to (Johan et al., 2013), the steel, metal, and rotating machinery often cause a path loss as high as $30-40 \mathrm{~dB}$. This jeopardizes stable wireless connection of personnel and machines on the shop floor or in the warehouse. Consequently, only one coverage layer provided by the existing wireless network planners (Liao et al., 2011a; Liao et al., 2011b) is vulnerable to these shadowing effects.

Furthermore, large-scale industrial WLAN (IWLAN) deployment has rarely been investigated in literature. For instance, the warehouse of a typical car manufacturer in Belgium measures $83,000 \mathrm{~m}^{2}$. If the grid size is one meter, there are then 83,000 candidate locations for placing an AP. Most of the wireless network planning research is only limited in a small- or medium-scale environment, varying from several hundreds of square meters to several thousands of square meters (Liu et al., 2015b). A large building floor of 12,600 $\mathrm{m}^{2}$ was considered in (Jaffres-Runser et al., 2006) for WLAN planning. But only 258 candidate AP locations and a dozen of APs were involved, which significantly reduces the complexity of the wireless planning problem. A similar simplification can be observed in (Abdelkhalek et al., 2015; Liu, 2015; Mukherjee et al., 2011), which only enables optimization at a small or medium scale. It is challenging to perform large-scale optimization because of the significantly increased computational resources (memory and CPU time) and the stricter requirement for efficient algorithm design.

Additionally, recent investigates focus on WSN planning rather than on WLAN planning, though the deployment cost of a dense WLAN is far more than ignorable. For example, a WSN often contains 10 - 1000 cheap sensor nodes 
Table 1

Review of recent wireless network planning studies

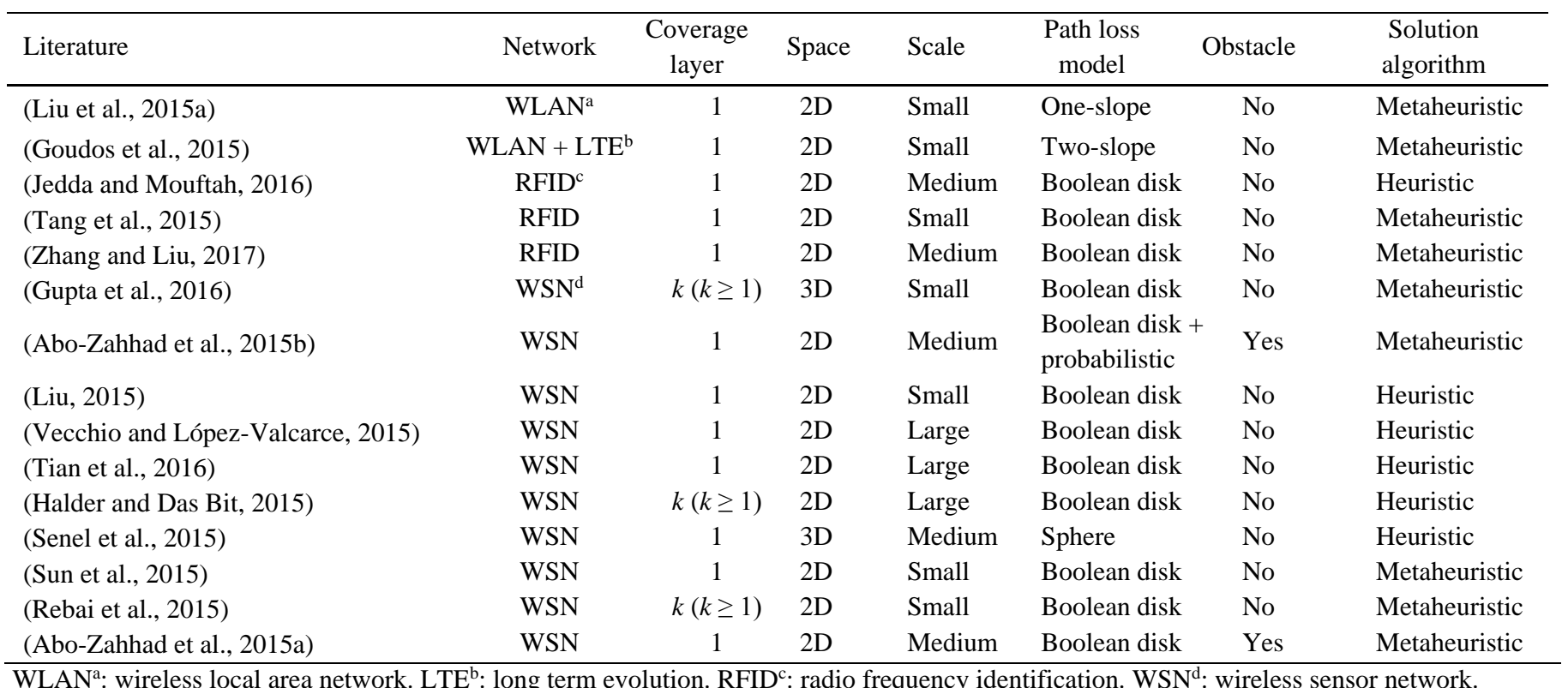

WLAN": wireless local area network. LTE $^{\mathrm{b}}$ : long term evolution. RFID ${ }^{\mathrm{c}}$ : radio frequency identification. WSN ${ }^{\mathrm{d}}$ : wireless sensor network.

(Mukherjee et al., 2011). If one sensor costs $10 €$, the deployment cost can surpass 10,000 €. As a result, the largescale property of a WSN makes it still of economic importance to perform WSN planning (Gupta et al., 2016; Liu, 2015; Rebai et al., 2015). Analogously, the deployment cost of a dense IWLAN is far more than ignorable due to the much higher price of an industrial AP and the large size of an industrial environment, though the number of APs would be smaller due to the larger coverage radius of an AP. For instance, the total cost of one Siemens ${ }^{\circledR}$ Scalance W788-2 M12 AP is more than $1550 €$, including the necessary accessories such as six antennas, one power cable, one power supply box, one connector, etc. Then the deployment of 100 these APs will cost more than 155,000 €, without even considering the labor cost and other engineering costs. Therefore, it is also of economic significance to minimize the IWLAN deployment cost.

This significance is even enhanced when redundant APs are deployed for enhancing robustness, which is a prevalent WSN deployment strategy (Chen et al., 2015). The idea of creating redundancy for reliable communications can be found in many other existing communication and network technologies, such as redundant radio (Park, 2016), multiple channels (Lin et al., 2010), and multiple network paths (Rizzi et al., 2016). A number of recent studies on WSN planning also create at least one redundant coverage layer for reliability (Gupta et al., 2016; Rebai et al., 2015). However, this idea is rarely observed in deploying redundant APs for a robust WLAN. Compared to most of existing work, the robustness enhancement approach proposed in this paper does not require any change in existing protocols, making it cost effective by using commercial off-the-shelf devices (i.e., APs).

Table 1 further summarizes and compares the recent studies on wireless network planning, in terms of network type, number of coverage layer, spatial dimensionality, problem size/scale, coverage/path loss model, consideration of obstacles, and solution algorithms. A fundamental issue for all these studies is coverage maximization, although the investigated problem differs in every study. Moreover, metaheuristics gain more popularity compared to heuristics that 
are very problem-dependent. As clearly presented gaps, most studies are limited within WSN planning, one coverage layer, 2D space, a small or medium problem size, a Boolean disk model, and ignorance of obstacles in the target environment. Though obstacles are considered in (Abo-Zahhad et al., 2015a; Abo-Zahhad et al., 2015b), the obstacle shadowing effect is simply modeled: a grid point is not considered to be covered by a sensor node if an obstacle is located between this sensor node and this grid point. Two more advanced path loss models are used in (Goudos et al., 2015; Liu et al., 2015a). But they neglect the shadowing of obstacles, due to their focus on office environments which are less harsh than industrial environments.

To fill these gaps, this paper makes fourfold contributions. (1) An industrial over-dimensioning (OD) problem is investigated. In this novel problem, two full WLAN coverage layers are planned in a large harsh industrial indoor environment, while the deployed cost is minimized. An empirical one-slope path loss model, which considers the shadowing effects of three-dimensional (3D) obstacles, is utilized for precise yet simple coverage calculation. (2) An efficient genetic algorithm based OD (GAOD) algorithm is proposed for solving this OD problem even at a large size. To enable large scale optimization, the solution representation, population initialization, crossover, and mutation of GAOD are designed, and parallel genetic search framework and problem-dependent speedup measures are proposed. (3) A greedy heuristic based OD (GAOD) is also introduced, which represents a state-of-the-art OD heuristic and serves as a benchmark algorithm for the GAOD. (4) The effectiveness and superiority of this GAOD is both experimentally validated and numerically demonstrated, in contrast to most wireless planning literature that only has numerical experiments without any real system deployment.

The rest of this paper is organized as follows. Sect. 2 mathematically formulates the OD problem. Sect. 3 defines the concepts that are frequently used in solution algorithm design. Sect. 4 and Sect. 5 presents the GHOD and the GAOD, respectively. Sect. 6 experimentally validates the proposed GAOD. Sect. 7 numerically demonstrates the effectiveness and efficiency of GAOD in two vehicle manufacturers' indoor environments, standing for a small and large industrial indoor environment, respectively. Sect. 8 draws conclusions.

\section{Over-dimensioning model}

The OD problem is to minimize the number of deployed industrial APs, under the constraints of two full coverage layers in a target industrial indoor environment and an inter-AP separation longer than a limit distance. Signal blockage caused by dominant 3D obstacles are considered in the path loss calculation. APs are assumed to be of the same type for homogeneous WLAN planning. A solution to the OD problem is denoted by $\vec{l}$, a vector of AP 2D locations.

The second coverage layer serves as redundancy against coverage holes on the first layer that are caused by dominant obstacles. If two or more APs are placed very close to each other, they are likely to be simultaneously shadowed by the same obstacle. To make the OD solution $\vec{l}$ robust against shadowing effects of obstacles, a minimal inter-AP separation $\left(d_{A P \min }\right)$, i.e., the minimal distance between any two APs, is thus a necessary and practical constraint for this OD problem. 
Table 2

Over-dimensioning model notation

\begin{tabular}{|c|c|c|c|}
\hline Symbol & Meaning & Symbol & Meaning \\
\hline AP & Access point & $P_{i j}$ & $\begin{array}{l}\text { Stable power received by the } i \text {-th grid point from the } \\
j \text {-th access point at } 99 \% \text { of the time }\end{array}$ \\
\hline$d_{A P \min }$ & Minimal separation distance of two arbitrary access points & $P L 0$ & $\begin{array}{l}\text { Path loss }(\mathrm{dB}) \text { at the location that is one meter away } \\
\text { from a target access point }\end{array}$ \\
\hline$d_{i j^{\prime}}$ & Distance between two access points & $P L\left(d_{i j}\right)$ & $\begin{array}{l}\text { Path loss (dB) between the } i \text {-th grid point and the } j \text { - } \\
\text { th access point }\end{array}$ \\
\hline$d_{\max }$ & Maximal coverage radius of an access point & $\mathrm{Rx}$ & Wireless signal receiver \\
\hline$G$ & Total gain of a pair of transmitter and receiver & Tx & Wireless signal transmitter \\
\hline GP & Grid point & $T H L D$ & Threshold received power $(\mathrm{dBm})$ of a client receiver \\
\hline$g p_{g}$ & The $g$-th grid point in an environment & $T P_{\max }$ & Maximal transmit power $(\mathrm{dBm})$ of an access point \\
\hline$g s$ & Basic grid size in a discretized environment & $x_{j}$ & Horizontal coordinate of an access point \\
\hline$I$ & Set of indices of grid points & $x \operatorname{Max}$ & Maximal horizontal coordinate of an environment \\
\hline$J$ & Set of indices of access points & $x$ Min & Minimal horizontal coordinate of an environment \\
\hline$\vec{l}$ & Location vector of over-dimensioned access points & $y_{j}$ & Vertical coordinate of an access point \\
\hline$M$ & $\begin{array}{l}\text { Margin }(\mathrm{dB}) \text { considering shadowing, fading, and } \\
\text { interference }\end{array}$ & $y \operatorname{Max}$ & Maximal vertical coordinate of an environment \\
\hline$n$ & Path loss exponent & $y$ Min & Minimal vertical coordinate of an environment \\
\hline$N_{A P}$ & Total number of over-dimensioned access points & $\Omega$ & Set of all grid points in an environment \\
\hline$N_{o}$ & Total number of dominant obstacles in an environment & $\xi$ & $\begin{array}{l}\text { Deviation between measurement and a path loss } \\
\text { model, with zero mean and a standard deviation } \sigma\end{array}$ \\
\hline$O L_{i j}$ & $\begin{array}{l}\text { Total obstacle loss (dB) between the } i \text {-th grid point and the } \\
j \text {-th access point }\end{array}$ & $\alpha_{i j}$ & $\begin{array}{l}\text { Logical coverage variable for the } i \text {-th grid point and } \\
\text { the } j \text {-th access point }\end{array}$ \\
\hline$O L_{k}$ & $\begin{array}{l}\text { Obstacle loss }(\mathrm{dB}) \text { of the } k \text {-th dominant obstacle in the } \\
\text { environment }\end{array}$ & $\beta_{i j}^{k}$ & $\begin{array}{l}\text { Logical signal blockage variable for the } i \text {-th grid } \\
\text { point, the } j \text {-th access point, and the } k \text {-th dominant } \\
\text { obstacle }\end{array}$ \\
\hline
\end{tabular}

In the following subsections, this OD problem is mathematically modeled in Sect. 2.1. A concise illustration is demonstrated in Sect. 2.2 for enhancing the ease of understanding of this OD model. Finally, the complexity analysis is performed in Sect. 2.3, in terms of time complexity of this model and its variables that influence the difficulty of large-scale optimization of such as OD problem.

\subsection{Model formulation}

Table 2 lists the notation of the proposed OD model. In this model, a target rectangular environment is $2 \mathrm{D}$, i.e., horizontal and vertical. It is represented by two extreme 2D points, i.e., the bottom left point ( $x$ Min, $y$ Min) and the top right $(x \operatorname{Max}, y$ Max). It is discretized into $g s \times g s$ small grids, where $g s$ is the grid size that is preset as an input of the model. Each grid point (GP) is represented by the upper-left vertex, and denoted as $g p_{g}$, where $g$ is a unique index for each GP. A lexicographical order is applied to all the GPs, i.e.,

$$
(x 0, y 0)<(x 1, y 1) \Leftrightarrow x 0<x 1 \vee(x 0=x 1 \wedge y 0<y 1)
$$


where $(x 0, y 0)$ and $(x 1, y 1)$ are two distinct GPs in the environment.

A target environment is thus described by a set of ordered GPs, which is denoted as $\Omega$. The GP index $i$ within $\Omega$ starts from one, corresponding to the extreme point ( $x$ Min, yMin) of the rectangular environment. It increases one by one following the lexicographical order, until reaching $|\Omega|$, the total number of GPs. Then the set of GPs is denoted by $I=\{1,2, \ldots,|\Omega|\}$. The following formula is used to determine the size of $\Omega$ :

$$
|\Omega|=\operatorname{ceil}[(x \operatorname{Max}-x \operatorname{Min}) / g s] \times \operatorname{ceil}[(y \operatorname{Max}-y \operatorname{Min}) / g s]
$$

A receiver $(\mathrm{Rx})$ is placed on each GP except the GPs where APs are placed. The received power on the downlink is considered in coverage calculation. Different physical bitrates of an Rx require distinct lowest received power levels, named threshold (THLD) hereafter. The quantified relation can be found in (Gong et al., 2016b). A GP is covered by an AP if the received power of the Rx on that GP is higher than or equal to the threshold. The coverage of an AP is hence represented as the GPs that are covered by this AP.

The maximal transmit power $T P_{\max }$ of an AP is considered, which is a natural way to help minimizing the number of over-dimensioned APs. All APs are of the same type. They can be placed anywhere within the target environment. Three assumptions are further made on the environment. (1) It has no previously installed APs. (2) It is so large that multiple APs are needed for even one complete layer of coverage. (3) It is empty and the shape is convex such that there is always the line-of-sight propagation between two locations. These assumptions are reasonable, since a shop floor and a warehouse are often a large rectangular hall. The shadowing effects of dominant obstacles are considered in the path loss calculation. There are $|\vec{l}|$ APs in an OD solution $\vec{l}$. The AP set is denoted as $J=\{1,2, \ldots,|\vec{l}|\}$, also following a lexicographical order.

Without loss of generality, a one-slope path loss model (Tanghe et al., 2008) is used to calculate power loss between the AP transmit power and the received power of an Rx, additionally considering the signal blockage caused by dominant obstacles:

$$
P L\left(d_{i j}\right)=P L 0+10 n \log _{10}\left(d_{i j}\right)+O L_{i j}+\xi
$$

where $P L 0$ (in $\mathrm{dB}$ ) is the path loss at the distance of one meter, $n$ is the path loss exponent which is a dimensionless parameter indicating that the path loss increases with the distance, $d_{i j}$ is the distance (in $\mathrm{m}$ ) between the Rx placed on the $i$-th GP and the $j$-th AP, $O L_{i j}$ is the total obstacle loss (in $\mathrm{dB}$ ) caused by the dominant obstacles that block the line between the Rx placed on the $i$-th GP and the $j$-th AP, and $\xi$ (in $\mathrm{dB}$ ) is the deviation between the measurement and model, which is attributable to shadowing.

Obstacle locations are assumed fixed in an environment. The deviation $\xi$ in Eq. (3) follows a Gaussian distribution, with a mean of zero and a standard deviation $\sigma$. The gain and margin are considered in the link budget calculation to be more realistic, which is not taken into account in (Siomina et al., 2007). The total gain $G$ (in $\mathrm{dB}$ ) is the sum of the AP transmitter's gain and the Rx's gain. The margin $M$ (in $\mathrm{dB}$ ) is the sum of shadowing margin, fading margin and interference margin. 


$$
\text { objective: } \min _{\left(x_{j}, y_{j}\right) \in \Omega, j \in J}(|\vec{l}|)
$$

s. t.:

$$
\begin{aligned}
& \sum_{j=1}^{N_{A P}} \alpha_{i j} \geq 2, \forall i \in I \\
& d_{\max }=10\left(\frac{T P_{\max }+G-M-T H L D-P L 0}{10 n}\right) \\
& O L_{i j}=\sum_{k=1}^{N_{o}} \beta_{i j}^{k} \cdot O L_{k}, \forall i \in I, \forall j \in J \\
& P_{i j}=T P_{\max }+G-M-P L\left(d_{i j}\right), \forall i \in I, \forall j \in J \\
& \alpha_{i j}=\left\{\begin{array}{l}
1, \text { if } P_{i j} \geq T H L D \\
0, \text { otherwise }
\end{array}, \forall i \in I, \forall j \in J\right. \\
& \beta_{i j}^{k}=\left\{\begin{array}{l}
1, \text { if the } k \text {-th obstacle blocks between the } i \text {-th GP and the } j \text {-th AP } \\
0, \text { otherwise }
\end{array}, \forall i \in I, \forall j \in J, \forall k \in\left\{0,1, \ldots, N_{o}\right\}\right. \\
& d_{j j^{\prime}} \geq d_{A P \min }, \forall j, j^{\prime} \in J, j \neq j^{\prime} \\
& 0<d_{A P \min }<d_{\max } / 2 \\
& x \operatorname{Min} \leq x_{j} \leq x \operatorname{Max}, y \operatorname{Min} \leq y_{j} \leq y \operatorname{Max}, \forall j \in J
\end{aligned}
$$

The OD model is formulated in Eqs. (4-13). Eq. (4) is the objective function for OD. The objective is to minimize the number of APs $(|J|)$ that are over-dimensioned. In homogeneous network planning, this equals minimization of deployment cost. The variable is the $2 \mathrm{D}$ location of each AP. The output of this objective function is a vector of APs that are over-dimensioned in a target industrial indoor environment. The number of AP is unknown and can vary between different solutions for the same OD problem. The rest formulations define the constraints of this OD problem.

Eq. (5) requires that each GP should be covered by at least two APs. Eq. (6) calculates the maximal distance $d_{\max }$ (in $\mathrm{m}$ ) that an AP can potentially cover, by considering the maximal transmit power on the AP side and the minimal received power (or THLD) on the Rx side, and without any obstacle blocking the line of this Tx-Rx pair. Eq. (7) calculates the total obstacle loss (in $\mathrm{dB}$ ) for the pair of $i$-th GP and $j$-th AP.

Eq. (8) calculates the power that an Rx on the $j$-th GP receives from the $i$-th AP. Eq. (9) defines the logical coverage variable $\alpha_{i j}$ for all pairs of GP-AP. It is one, if the power that an Rx on the $i$-th GP receives from the $j$-th AP is not lower than THLD. Otherwise, it is zero. Eq. (10) defines the logical blockage variable $\beta_{i j}^{k}$ for the $i$-th GP, $j$-th AP, and $k$-th dominant obstacle. It equals one, if the $k$-th dominant obstacle blocks the line-of-sight propagation between the $j$ th AP and $i$-th GP. Otherwise, it equals zero. 


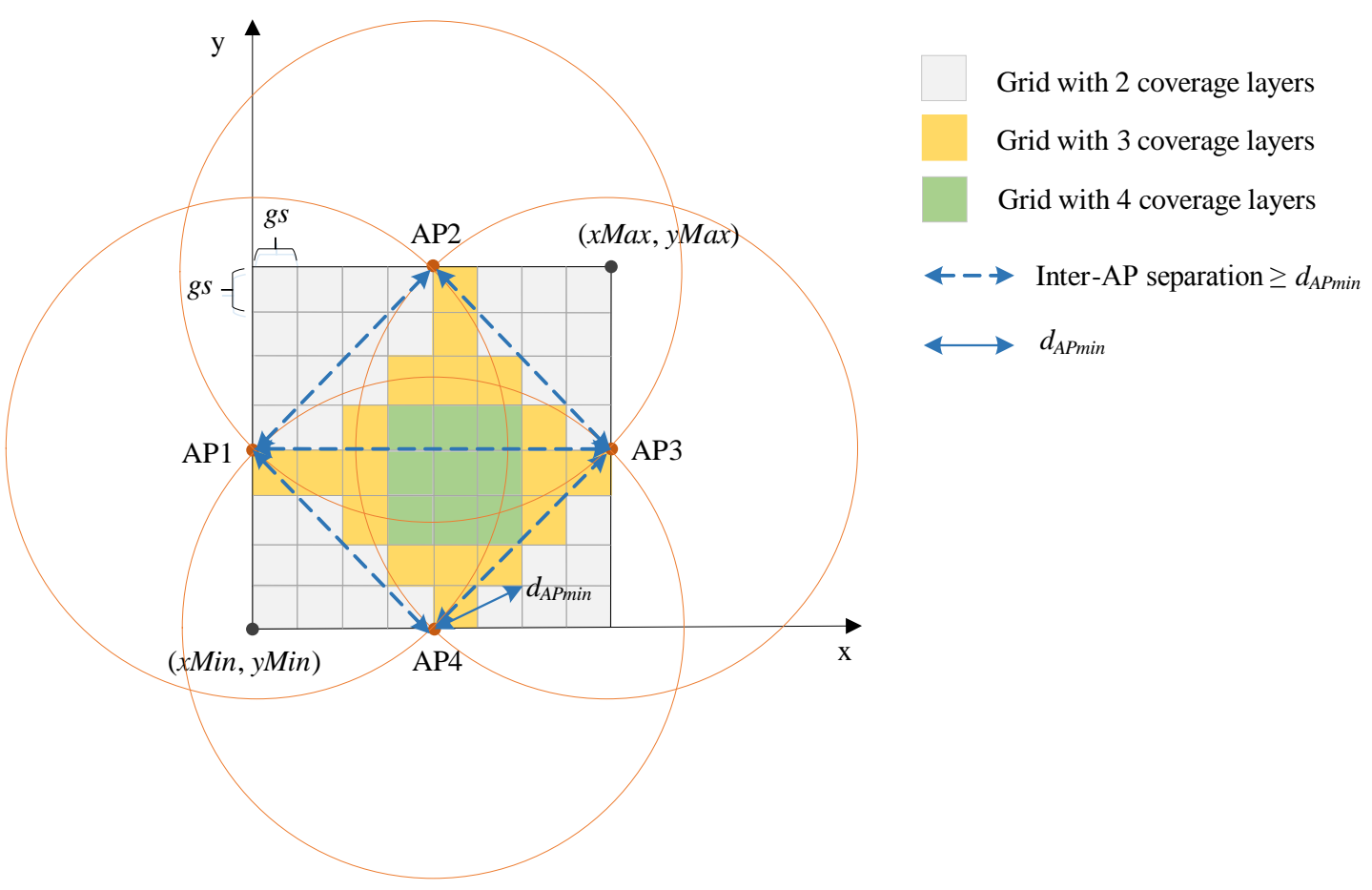

Fig. 1. Illustration of an over-dimensioning (OD) model: 4 access points (APs) are placed in the target environment such that each $g s \times g s$ grid is covered at least twice and any two APs should not be placed within a minimal spatial distance $d_{A P \min }$. A smaller number of APs indicates high quality of an OD solution.

Eq. (11) forces that any intra-AP separation should not be shorter than the preset limit distance $d_{A P \min }$. Eq. (12) sets the lower and upper bounds of $d_{A P \min }$. Eq. (13) indicates where APs can be placed: inside the rectangle target environment or exactly on the boundaries (i.e., side walls of an industrial indoor environment).

\subsection{Illustration of an over-dimensioning model}

The formulated OD model is illustrated by a simple example. As depicted in Fig. 1, a target rectangular environment is characterized by its bottom-left vertex ( $x$ Min, yMin) and top-right vertex ( $x$ Max, yMax). It is discretized into $8 \times 8$ grids. Each grid is sized by $g s \times g s$. If the top-left vertex of a grid is within the coverage of an AP, this grid is considered covered by this AP. The demonstrated OD solution in Fig. 1 is qualified, in the sense that all grids are covered at least twice, and any pair of APs are placed beyond the required minimal separation distance $d_{A P m i n}$. Evidently, a new OD solution is also qualified if additional APs are placed in Fig. 1 while satisfying the inter-AP separation of $d_{A P m i n}$. However, this new OD solution is inferior, as it outputs more APs than the OD solution shown in Fig. 1.

Fig. 2 further illustrates how to determine an Rx, which is placed on a grid, is covered by an AP. Fig. 2(a) presents the top view. Rx1 and Rx3 are covered by an AP, as they are within the maximal coverage radius $\left(d_{\max }\right)$ of this AP. Moreover, Rx1 receives a stronger signal than Rx3, as Rx1 is closer to the AP. Comparatively, Rx2 is not covered due to the significant shadowing effect of the obstacle in-between the AP and Rx2. Rx4 is not covered by the AP, either, as it is located beyond $d_{\max }$. Fig. 2(b) shows the side view of the same coverage. Although both R2 and R3 are behind the obstacle in the two horizontal dimensions in Fig. 2(a), R3 is not vertically within the shadow of this obstacle. 


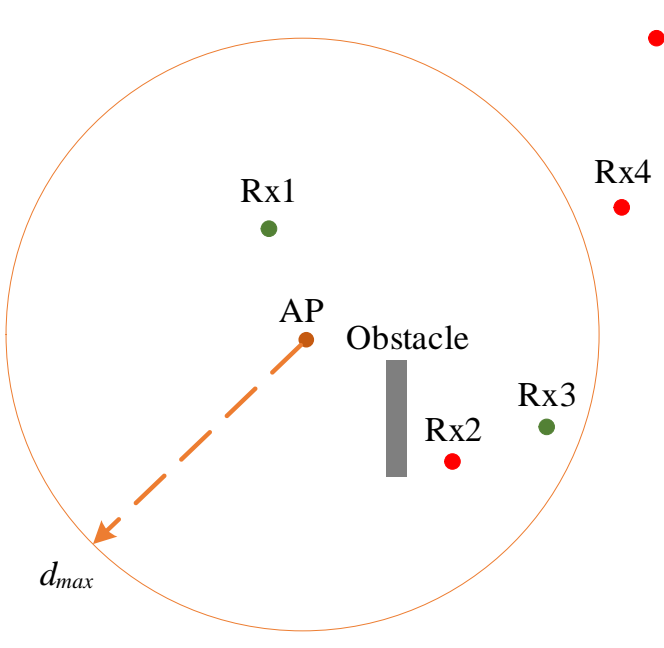

(a) Top view of the coverage of an AP
- Rx that is not covered $\mathrm{Rx}$ that is covered Obstacle shadow

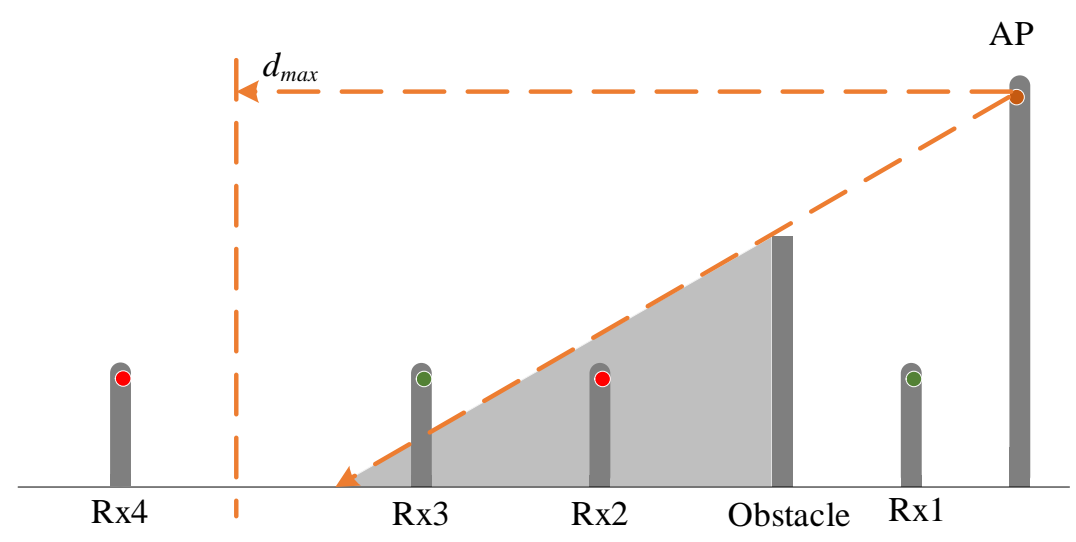

(b) Side view of the coverage of an AP

Fig. 2. Illustration of the three-dimensional coverage of an access point (AP). The maximal coverage radius of this AP is $d_{\max }$.

Consequently, R3 is still covered by the AP, while Rx 2 is shadowed by the obstacle whose signal loss is strong enough to make Rx2 uncovered by the AP.

\subsection{Complexity analysis}

As shown in (Ke et al., 2011; Ke et al., 2007), it is non-deterministic polynomial complete (NP-complete) to achieve $k$-cover with minimum of nodes in grid-based networks. Complying with this condition, the above OD problem has additional constraints of 3D obstacle shadowing and AP separation. Therefore, this OD problem is NP-complete.

According to the classification of difficult factors in large-scale optimization (Omidvar et al., 2015), the complexity of a large-scale OD problem is influenced by the following variables: (1) the size of an industrial indoor environment, (2) the number of APs, (3) the spatial resolution (grid size or $g s$ ), (4) the number of coverage layers, (5) the spatial separation of APs or interdependency of AP placements, (6) the path loss model that is used. The first three variables determine the size of a search space, i.e., the total number of possible solutions. On the one hand, the fourth and fifth variables add additional yet practical constraints, which change the property of a search space. One the other hand, they introduce the active interaction between AP locations, which means that the location of each AP cannot be individually determined to find the global optimal solution. The last variable impacts the expense of evaluating a solution during which the path loss between AP and an Rx is extensively calculated.

Given the constraint of double full coverage and minimal AP separation, the number of APs highly depends on the size of an industrial indoor environment and the spatial resolution $(g s)$. Therefore, an OD problem is considered as large-scale if the target industrial indoor environment has a large size (> $\left.10000 \mathrm{~m}^{2}\right)$ and $g s$ is small (within several meters). Otherwise, it is considered as small-scale. 


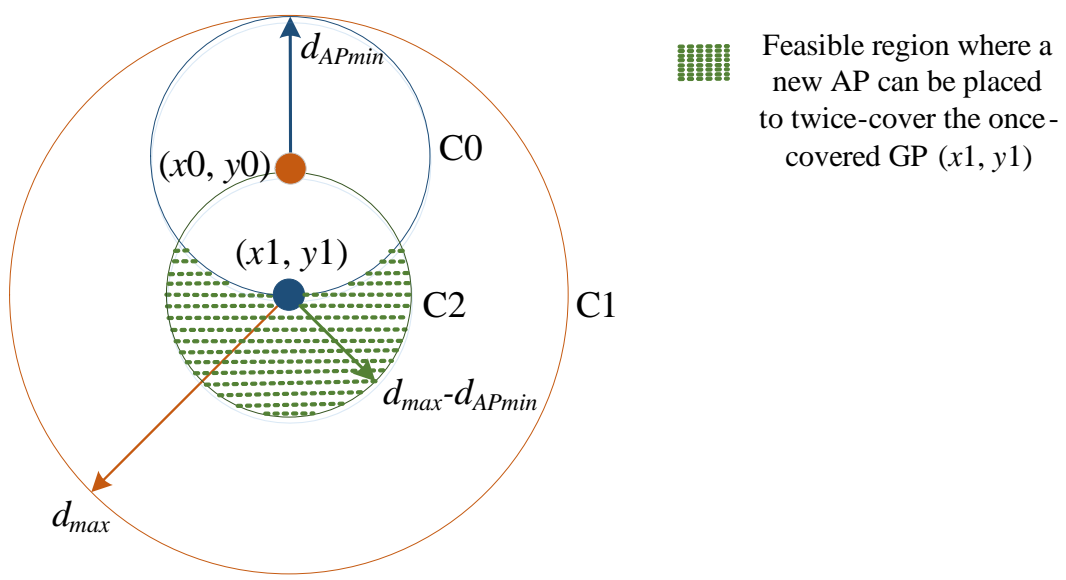

Fig. 3. Demonstration of double full coverage in an extreme case: $(\mathrm{x} 0, \mathrm{y} 0)$ and the outer edge of circle $\mathrm{C} 1$ are placed with APs, such that $(\mathrm{x} 1, \mathrm{y} 1)$ is only once-covered and only the shadowed region, i.e., $\mathrm{C} 2-\mathrm{C} 0 \cap \mathrm{C} 2$, is feasible for accommodating an additional AP to twice cover (x1, y1).

\section{Concept definitions for over-dimensioning algorithmic design}

Several frequently-used concepts and properties are defined to facilitate the design of an OD solution algorithm in the following Sects. $4 \& 5$.

Definition 1: covered GPs refer to the set of GPs that are covered by at least two APs at the maximal transmit power level.

Definition 2: once-covered GPs stand for the set of GPs that are covered by only one AP.

Definition 3: new once-covered GPs represent the set of GPs that are not yet covered by any AP, but can be covered by a given AP at the maximal transmit power level.

Definition 4: new twice-covered GPs denote the set of GPs that are covered only once, and can be covered twice by a given AP at the maximal transmit power level.

Definition 5: candidate GPs indicate the set of GPs that are available for placing APs.

Definition 6: uncovered GPs refer to the set of GPs that are covered less than twice by all placed APs at the maximal transmit power level.

Definition 7: valid GPs refer to the set of GPs that are located beyond the minimal AP separation $\left(d_{A P m i n}\right)$ of all the APs that are already placed in the environment.

Theorem 1: by placing APs of the same type (i.e., equal $d_{\max }$ ) one by one, all the GPs within $d_{A P \min }$ of all placed APs can be covered at least twice.

Proof: an extreme case is assumed (Fig. 3). The purpose is to find in such an extreme case a feasible region for accommodating a new AP to twice-cover the once-covered GP $(x 1, y 1)$. As $(x 1, y 1)$ is covered by an AP on $(x 0, y 0)$, circle0 (C0) can be obtained: $(x-x 0)^{2}+(y-y 0)^{2}=d_{A P \min }{ }^{2}$. Given the constraint of minimal AP separation $\left(d_{A P \min }\right)$ defined by Eq. (11), no additional AP can thereby be placed within C0. Besides, existing APs are already placed on 


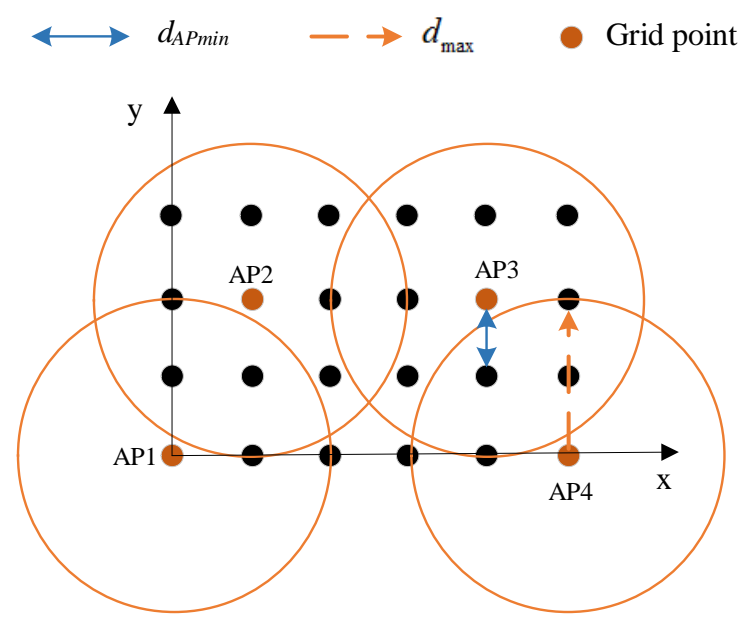

(a) First full coverage layer

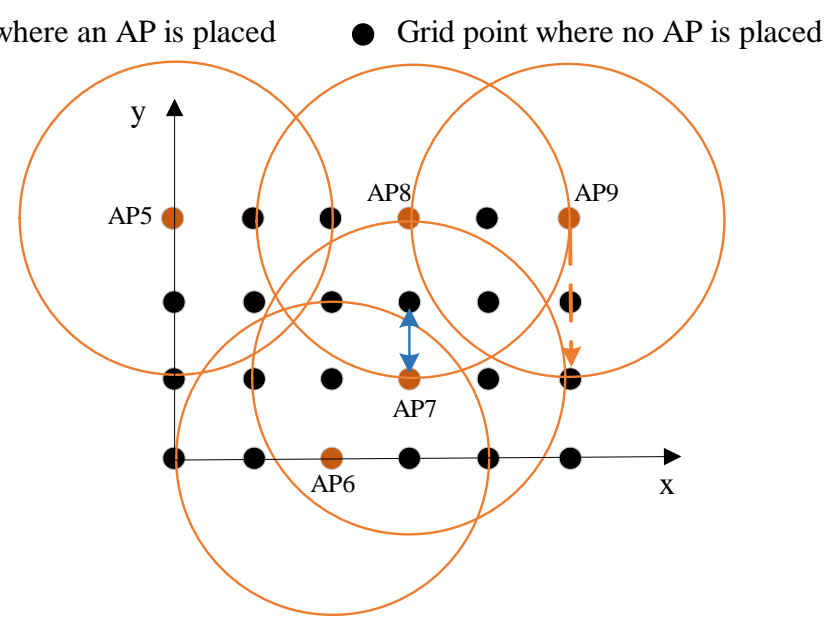

(b) Second full coverage layer

Fig. 4. Full double coverage over the area beyond the minimal AP separation distance for the case where $d_{A P \min }=d_{\max } / 2=g s$ (grid size). Large orange circle denotes the coverage area of an AP with the maximal coverage radius $d_{\max }$.

the outer edge of circle1 (C1): $(x-x 1)^{2}+(y-y 1)^{2}=d_{\max }{ }^{2}$, exerting extra constraints of $d_{A P \min }$ on the feasible region. Consequently, the only feasible region is $\mathrm{C} 2-\mathrm{C} 0 \cap \mathrm{C} 2$, i.e., the shadowed region in Fig. 3, where circle2 (C2) is $(x-x 1)^{2}+(y-y 1)^{2}=\left(d_{\max }-d_{A P \min }\right)^{2}$. This feasible region always exists due to the lower and upper bounds of $d_{A P \min }$ defined by Eq. (12), such that $d_{\max }-d_{A P \min }>0$. As a result, the once-covered GP on $(x 1, y 1)$ can always be twicecovered, by placing a new AP in this feasible region. Therefore, theorem 1 is true in this extreme case. In addition, many less stringent cases evidently exist which fit theorem 1. For instance, if not all the GPs on the outer edge of $\mathrm{C} 1$ are placed with APs in Fig. 3, the feasible region to place a new AP obviously becomes larger. Furthermore, if multiple once-covered GPs exist regardless of the location within $\mathrm{C} 0$, the aforementioned process can be iterated. Therefore, theorem 1 remains true.

Theorem 2: by placing APs of the same type (i.e., equal $d_{\max }$ ) one by one, all the GPs beyond $d_{A P \min }$ of all placed APs (i.e., valid GPs) can be covered at least twice.

Proof: given the constraint of Eq. (12), this property can be bounded by two extreme cases, i.e., $d_{A P \min }=0$ and $d_{A P \min }=d_{\max } / 2$. If $d_{A P \min }=0$, then two APs can be placed on the same GP for double full coverage of the same area. Theorem 2 is thus true. If $d_{A P \min }=d_{\max } / 2$, the environment is sure to have the first coverage layer, as illustrated in Fig. 4a where $d_{\max }=2 g s$ and the first full coverage layer can be formed up by AP1, AP2, AP3 and AP4. Then, the environment can be fully covered for the second time by five additional APs, as illustrated in Fig. 4b. Obviously, all the placed APs are beyond $d_{A P \min }(g s)$. Theorem 2 is thus true. There are many more cases between the two bounds that can meet with theorem 2 . Therefore, theorem 2 remains true. 


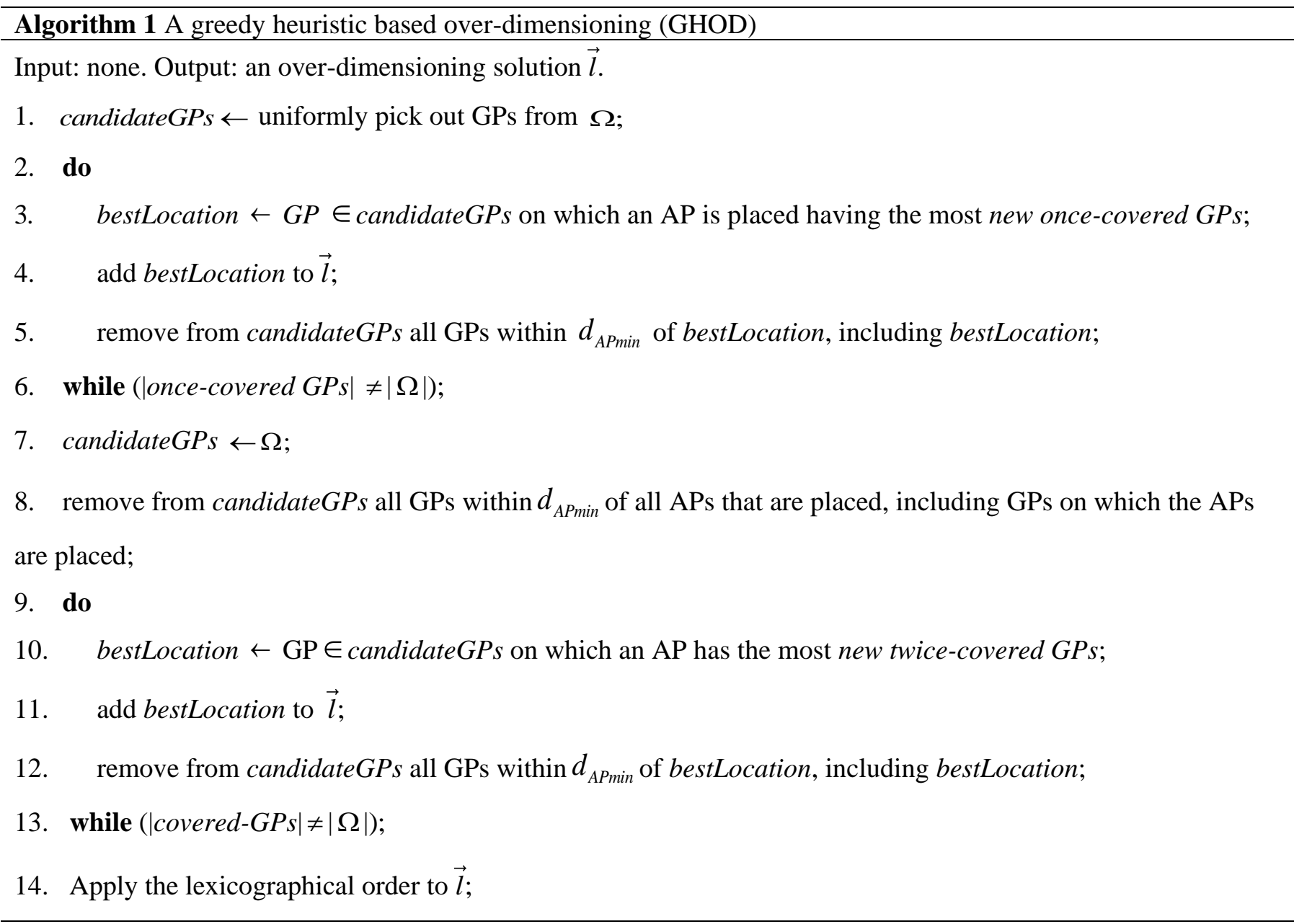

\section{Greedy heuristic based over-dimensioning (GHOD)}

The GHOD is proposed in this section. It represents a heuristic to solve the OD problem and can serve as a benchmark algorithm for the GAOD proposed later in Sect. 4. It is inspired from the recently proposed wireless planning algorithm in (Liu et al., 2015b). This original algorithm determines the minimal number of APs and their locations while satisfying a specified physical bitrate in an office environment. The same idea is used in the GHOD, by placing APs one after another such that a target environment is gradually covered toward the required coverage rate. Therefore, the GHOD represents a specific heuristic for this OD problem (Sect. 2).

However, two enhancements have been made to adapt to this OD problem. First, it creates two coverage layers instead of one and under the additional constraint of a minimal inter-AP separation. Second, it achieves the linear-time calculation in setting up the first coverage layer, by reducing the time complexity from $O\left(n^{3}\right)$ to $O(n)$, where $n$ is the size of a $2 \mathrm{D}$ environment.

The time complexity $O\left(n^{3}\right)$ in the original algorithm is introduced by the $\mathrm{d}_{\text {avg }}$ criterion in judging the best AP location when placing each AP. This criterion calculates the average distance among all uncovered GPs. It is effective to offer a minimized number of APs for a full coverage layer. Nevertheless, two full coverage layers should be set up in OD, 


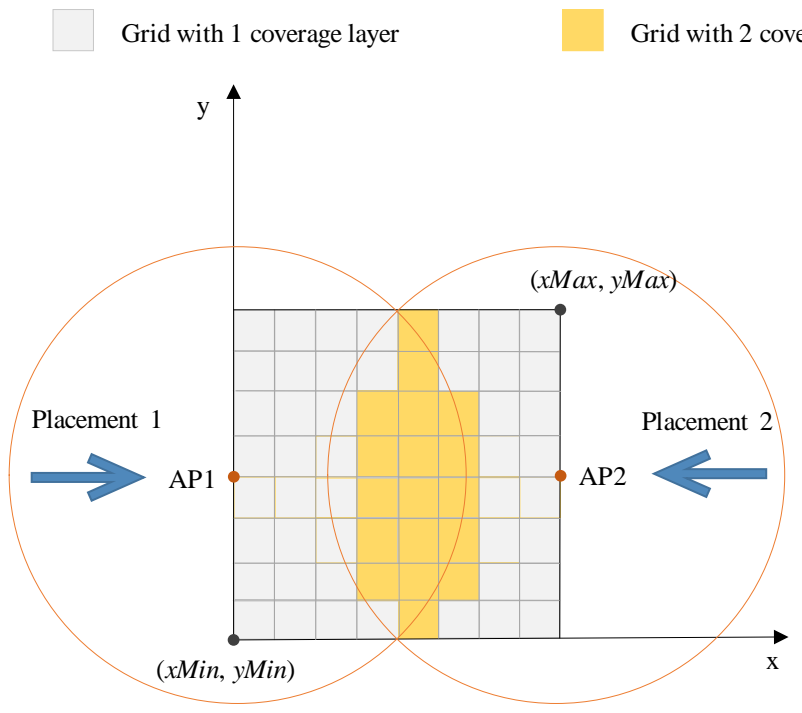

(a) AP1 and AP2 achieve the first full coverage layer

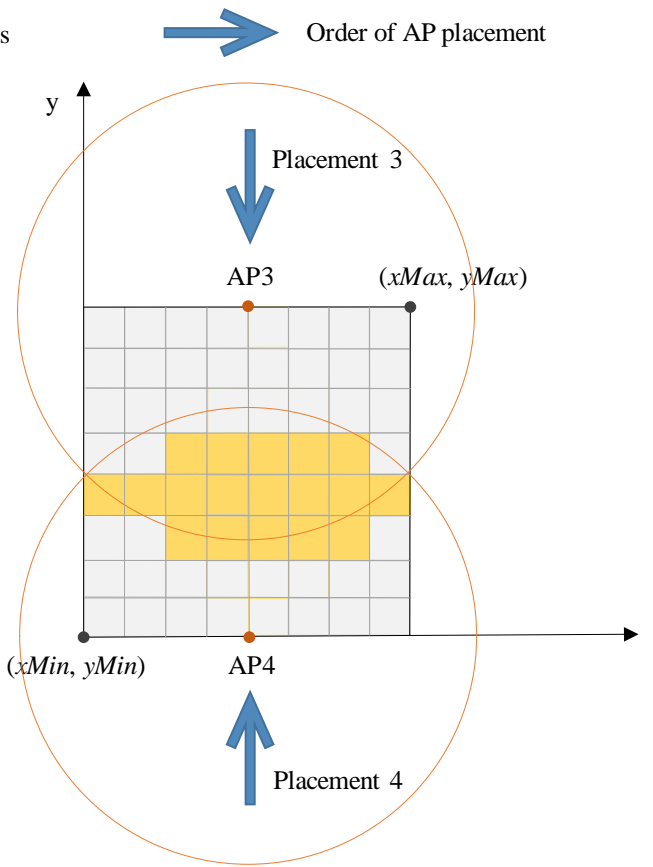

(b) AP3 and AP4 achieve the second full coverage layer

Fig. 5. Illustrative example of the greedy heuristic based over-dimensioning (GHOD). Access points (APs) are sequentially placed such that AP1 and AP2 ensure the first coverage layer, while AP3 and AP4 guarantee the second coverage layer. Therefore, these 4 APs achieve double full coverage layers in the target environment.

which changes the context of the $\mathrm{d}_{\mathrm{avg}}$ criterion. Besides, $O\left(n^{3}\right)$ is inefficient for large-scale network planning. For instance, it took $1093 \mathrm{sec}$ for setting up one full coverage layer over an environment of $200 \mathrm{~m} \times 50 \mathrm{~m}$ at a PC with an Intel i5-3470 CPU and 8G RAM. It will then take about 6.25E5 sec (about $173.6 \mathrm{~h}$ ) for an industrial hall of $415 \mathrm{~m} \times$ $200 \mathrm{~m}$, which is very time consuming for network planning. The original algorithm was actually run for the latter largesized problem. As expected, no result was obtained after $40 \mathrm{~h}$.

To reduce the time complexity, the criterion of determining the best AP in (Liu et al., 2015b) is altered in the proposed GHOD. As described in Algorithm 1, the GHOD establishes the two coverage layers one by one. Candidate AP locations are first established (line 1), and then iterated for picking out the best location (lines 2-6). A new AP is placed on the best location, and is powered on with the maximal transmit power to cover as many GPs as possible (line 3). The best location is the one on which a newly-placed AP contributes to the highest number of new once-covered GPs. It is then added to the OD solution vector $\vec{l}$ (line 4). After that, the candidate AP locations are updated by removing once-covered GPs as well as the new AP's location (line 5). The former process of AP placement (lines 3-5) is iterated until the environment is fully once-covered (line 6). Then a new set of candidate AP locations are created by removing all GPs that are within $d_{\text {APmin }}$ of existing APs including AP locations (lines 7-8). In this way, these new candidate AP locations can fully comply with the constraint of minimal AP separation distance, i.e. Eq. (11). Then an analog iteration is performed to ensure the environment is fully twice-covered (lines 9-13). APs in the final OD solution are reordered by following the lexicographical order (line 14), i.e., Eq. (1). Due to theorems $1 \& 2$, the two iterations (lines 2-6 \& 913, Algorithm 1) cannot be endless loops. Fig. 5 further gives an example to perform the GHOD. 


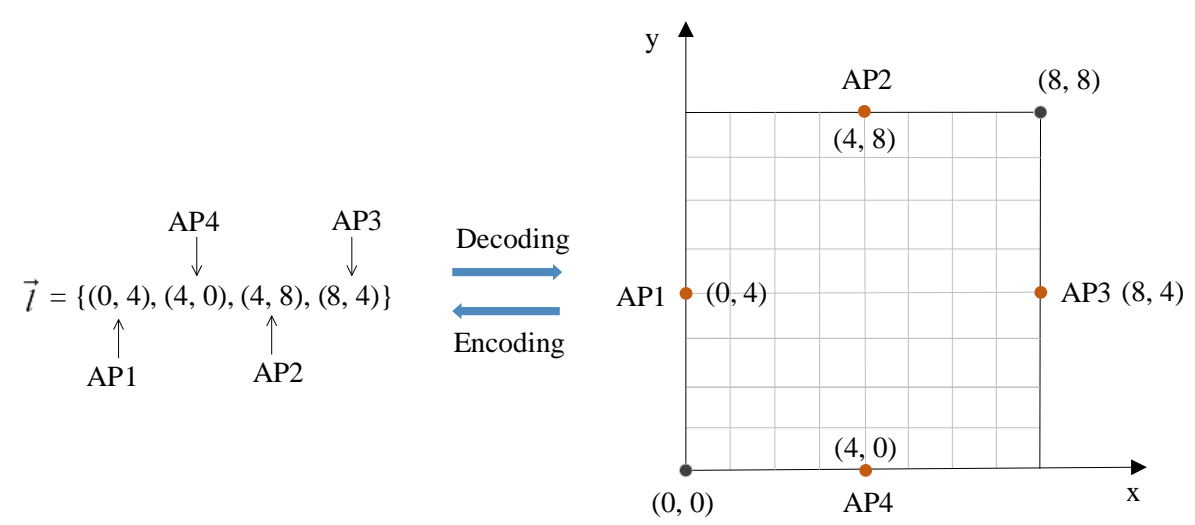

Fig. 6. Illustrative example of the solution encoding

\section{Genetic algorithm based over-dimensioning (GAOD)}

The GHOD algorithm treats an OD solution as sequential steps, and makes the local optimal decision at each step (Sect. 4). Although it has a simple time complexity of $O(n)$, it cannot guarantee a global optimal solution.

Comparatively, a genetic algorithm (GA) is one of the best-known metaheuristics in the family of evolutionary algorithms. It can give a global optimal or near-optimal solution within a reasonably short period. It has been successfully applied to solve planning and optimization problems for the manufacturing industry, such as energy-costaware production scheduling (Gong et al., 2016a). The GAOD is thereby proposed for the formulated OD problem (Sect. 2). To facilitate large-scale optimization, three aspects are specifically focused on in the design of GAOD, including enhancement of search efficiency, reduction of computational time, and decrease of temporary memory usage.

\subsection{Solution encoding}

It requires special concern on the solution encoding for an efficient genetic search. Authors in (Gupta et al., 2016) encode a wireless sensor placing solution as a vector, which contains all the candidate GPs for placing APs in a target environment. If a location is placed with a sensor, the value with the corresponding index in the vector is one. Otherwise, the value is zero. Unfortunately, enormous redundancy exists in this encoding space. It is natural that the number of candidate locations is larger than or equal to the number of wireless nodes to be placed. Therefore, the candidate locations, on which no AP is finally placed, contribute nothing to the final wireless planning solution. Consequently, redundancy exists in solution encoding space, which impedes the efficiency of a genetic search. A similar concern is described in (Yoon and Kim, 2013), where a normalization method is employed to map between the genotype space and the phenotype space.

To minimize memory usage and remove encoding redundancy, the proposed GAOD encodes an OD solution as a vector that only contains the 2D locations of over-dimensioned APs, i.e., the APs that are determined to be placed on a specified location in a target environment. This vector follows the lexicographical order in Eq. (1). Fig. 6 illustrates an example of such an encoding/decoding scheme: although APs are placed in the order of their indices (i.e., AP1, 
$\mathrm{AP} 2, \mathrm{AP} 3$, and AP4), they are reordered in the solution vector $\vec{l}$ by following the lexicographical order (i.e., AP1, AP4, AP2, and AP3). Each element in $\vec{l}$ denotes a 2D location in the target environment for the decoding process, and vice versa for the encoding process. Compared to conventional encoding schemes which require the length of a chromosome to be fixed, the proposed encoding scheme enables chromosomes to have varying lengths and thus varying dimensionality of a search space. Though this leads to light-weighted OD solution representation, the key genetic operators (initialization, crossover, and mutation) need to be specifically designed, which will be introduced in Sects. 5.2-5.5.

\subsection{Population initialization}

The initial population contains popSize qualified individuals which are randomly generated. The purpose is to guarantee that each initial individual can satisfy all the constraints of the OD model, and consequently to ensure an effective large-scale genetic research.

Generally, it is not a prerequisite to initialize individuals that fully satisfy the constraints. Individuals that cannot comply with all the constraints can be assigned with the worst fitness, and are likely to be eliminated by elitism and roulette wheel selection (Cheng et al., 2016). Besides, these worst individuals have the opportunity to be improved through crossover and mutation.

Despite the feasibility of the above disqualification handling method, it obviously wastes computational resources, by possibly having unqualified individuals as candidate solutions, and evolving based on a mix of qualified and unqualified individuals. As the efficiency of a genetic search is a sensitive factor for large-scale optimization, it is of crucial importance to remove an unqualified solution upon its appearance, such that no additional computational resources are needed to propagate this disqualification. To demonstrate that the propagation of disqualification significantly affects the efficiency of a large-scale genetic search, a computational experiment was conducted. In this experiment, more than 1000 random individuals were independently generated for a large-scale OD model (a warehouse of $415 \mathrm{~m} \times 200 \mathrm{~m}$ ). These individuals do not necessarily satisfy the constraint of two full coverage layers that are defined in Eq. (5). As a result, no qualified OD solution was obtained from this experiment. This thus highlights the necessity of a method that fully generates qualified solutions. Such a method is described in Algorithm 2 which produces a qualified initial individual.

Algorithm 2 comprises two sequential procedures after the initialization (lines 1-3): iteration 1 (lines 4-11) and iteration 2 (lines 12-26). The initialization allows the accommodating PC to temporally allocate memory to the three variables (i.e., validGPs, uncoveredGPs and candidateGPs), instead of storing them locally at each individual. This is because the number of GPs in an industrial indoor environment can be huge. It may take up a significant amount of memory to represent all the GPs. The genetic search may thereby suffer from a lack of memory.

Iteration 1 places APs one by one on GPs that are covered less than twice and beyond $d_{A P \min }$ of all APs that are already placed. It is greedy in the sense that the number of uncovered GPs decreases one loop after another. But it does not have to be strictly greedy as the GHOD, i.e., uncovered GPs do not have to reduce to the maximal degree in each 
Algorithm 2 Individual initialization of the genetic algorithm-based over-dimensioning (GAOD) Input: none. Output: an over-dimensioning solution $\vec{l}$.

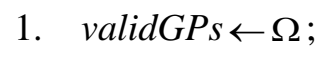

2. uncoveredGPS $\leftarrow \Omega$;

3. candidateGPs $\leftarrow \Omega$;

4. do

5. $\quad$ add to $\vec{l}$ a random GP of candidateGPs;

6. place a new AP on this GP and power it on with $T P_{\max }$;

7. remove from validGPs all GPs within $d_{A P \min }$ of this GP;

8. increase by one the coverage layer number of each GP that is within the coverage of this AP;

9. remove the new covered GPs from uncoveredGPs;

10. $\quad$ candidateGPs $\leftarrow$ validGPs $\cap$ uncoveredGPs;

11. while (candidateGPs $\neq \varnothing$ );

12. while (uncoveredGPs $\neq \varnothing$ )

13. centerGP $\leftarrow$ first GP in uncoveredGPs;

14. poolGPs $\leftarrow$ all GPs within the $d_{A P \min } \times d_{A P \min }$ square which is centered at centerGP;

15. candidateGPs $\leftarrow$ poolGPs $\cap$ validGPs;

16. if $($ candidate $\operatorname{los}==\varnothing)$

17. poolGPs $\leftarrow$ all GPs within the $d_{\max } \times d_{\max }$ square which is centered at centerGP;

18. $\quad$ candidateGPs $\leftarrow$ poolGPs $\cap$ validGPs;

19. end if

20. add to $\vec{l}$ a random GP of candidateGPs

21. remove from validGPs all GPs within $d_{A P \min }$ of this GP;

22. place a new AP on this GP and power it on with $T P_{\max }$;

23. increase by one the coverage layer number of each GP that is within the coverage of this AP;

24. remove the new covered GPs from uncoveredGPs;

\section{5. end while}

26. apply the lexicographical order to $\vec{l}$;

loop of iteration 1 . This is because this strict greedy property will not guarantee the global optimum while raising the computation burden. Furthermore, it cannot be endless owing to theorem 2 (Sect. 3). 
Iteration 2 (lines 12-26, Algorithm 2) follows and complements iteration 1. It intends to cover all the uncovered GPs,

which are located within $d_{A P \min }$ of the placed APs, in a number of loops. In each loop, candidate GPs are selected from a specified rectangular area, which is centered at the first GP of uncovered GPs (line 13). First, it is a small $d_{\text {APmin }} \times$ $d_{A P \min }$ rectangle (lines 14-15), such that a new AP can be placed in the vicinity of the first uncovered GP, while staying beyond $d_{A P \min }$ of all placed APs. If this small area has no qualified candidate GPs, a large $d_{\max } \times d_{\max }$ area is then created (lines 16-19). Given theorem 1 (Sect. 3), there must exist GPs within this large area that can accommodate new APs to cover the first uncovered GP for at least twice. Therefore, iteration 2 cannot be endless, either.

Moreover, as Algorithm 2 generates a random OD solution, it represents an ad hoc manual AP placement in practice and can be used as a benchmark for GHOD and GAOD. An individual is and OD solution. The numbers of APs in different individuals may differ due to the randomness of Algorithm 2. The minimization of AP number will depend on the population evolution, which is driven by crossover, mutation, and elitism.

\subsection{Crossover}

The one-point crossover operation, which guarantees the qualification of offspring for the OD problem, is defined by Algorithm 3. The input is two qualified individuals (i.e., indiv1 and indiv2 in Algorithm 3), which are selected by the roulette wheel selection mechanism (Cheng et al., 2016).

The crossover point is defined as a vertical line, named $x$ Crossover. The horizontal coordinate of $x$ Crossover is randomly selected (line 3) from the effective range calculated by lines 1-2 in Algorithm 3. This vertical line splits the rectangular environment into two rectangular subparts, i.e., the parts of which all the involved horizontal coordinates are smaller (part 1) and larger (part 2) than the randomly selected one, respectively. Then the two parts on the two individuals are swapped to get two children solutions (lines 4-6).

The constraint of minimal AP separation may be broken after the swap. However, it is unnecessary to check over the environment, since this can only occur within the small rectangular area around the vertical split line, i.e., $x$ Crossover $-d_{\text {APmin }} \leq x \leq x$ Crossover $+d_{\text {APmin }}$. Therefore, for speedup within this small rectangular area, if an AP is within $d_{A P \min }$ of another AP, this AP is removed from the OD solution represented by the current child (line 8).

The two children solutions are then checked (lines 9-13) whether they satisfy the constraint of two full coverage layers defined by Eq. (5). If this constraint cannot be satisfied, iterations 1 and 2 in Algorithm 2 will be performed (lines 14-22, Algorithm 3). This is not costly in terms of time and memory, since after a swap, this constraint can be broken only in the small area $x$ Crossover $-d_{A P \min } \leq x \leq x$ Crossover $+d_{A P \min }$.

To remain memory efficient, memory-consuming variables (such as uncoveredGPs and validGPs in Algorithm 3) do not have to be locally stored in the individuals and population. Instead, they are locally generated, meaning that the occupied huge memory will be immediately freed up at the end of Algorithm 3. 
\begin{tabular}{l} 
Algorithm 3 Crossover of the genetic algorithm-based over-dimensioning (GAOD) \\
\hline Input: $i n d i v l$ and indiv 2 Output:
\end{tabular} Input: indiv1 and indiv2. Output: newIndiv1 and newIndiv2.

1. $x$ Min $\leftarrow \max$ (minimal horizontal coordinates of all APs in indiv1 and indiv2);

2. $x \operatorname{Max} \leftarrow \min ($ maximal horizontal coordinates of all APs in indiv1 and indiv2);

3. $x$ Crossover $\leftarrow$ a random coordinate $\in[x \operatorname{Min}, x \operatorname{Max})$;

4. chop graphically indiv1 and indiv2 into two parts along the same vertical line $x$ Crossover, respectively

5. newIndiv $1 \leftarrow 1^{\text {st }}$ part of indiv $1+2^{\text {nd }}$ part of indiv 2 ;

6. $\quad$ newIndiv $2 \leftarrow 1^{\text {st }}$ part of indiv $2+2^{\text {nd }}$ part of indiv 1 ;

7. for indiv $\in\{$ newIndiv1, newIndiv 2$\}$

8. remove APs that are within $d_{A P \min }$ of any $\mathrm{AP} \in\{\mathrm{APs}$ in indiv $\}$ in the rectangular area

$x$ Crossover $-d_{A P \min } \leq x \leq x$ Crossover $+d_{A P \min }$

9. uncoveredGPs $\leftarrow \Omega$;

10. for $\mathrm{AP} \in\{\mathrm{APs}$ that remain in indiv $\}$

11. increase by one the coverage layer number of each GP within the coverage of the new AP;

12. remove all new covered GPS from uncoveredGPs;

13. end for

14. if (uncoveredGPs $\neq \varnothing$ )

15. validGPS $\leftarrow \Omega$;

16. for $\mathrm{AP} \in\{$ APs that remain in indiv $\}$

17. remove from validGPs all GPs within $d_{A P \min }$ of this AP;

18. end for

19. $\quad$ candidateGPs $\leftarrow$ validGPs $\cap$ uncoveredGPs;

20. iteration 1 (lines 4-11) in Algorithm 2;

21. iteration 2 (lines 12-25) in Algorithm 2;

22. end if

23. end for

24. apply the lexicographical order to newIndiv1 and newIndiv2;

\subsection{Mutation}

A mutation operation must produce a new qualified individual. This new individual should be different from all existing individuals as much as possible, because in concept mutation adds diversity to a generation and avoids a GA search to quickly converge in a single direction in the solution space.

To this end, Algorithm 4 is designed for mutation in the ODGA. It mainly consists of two steps. At step 1 (lines 110), additional APs of the same type are added to the target environment, while respecting the constraint of minimal 


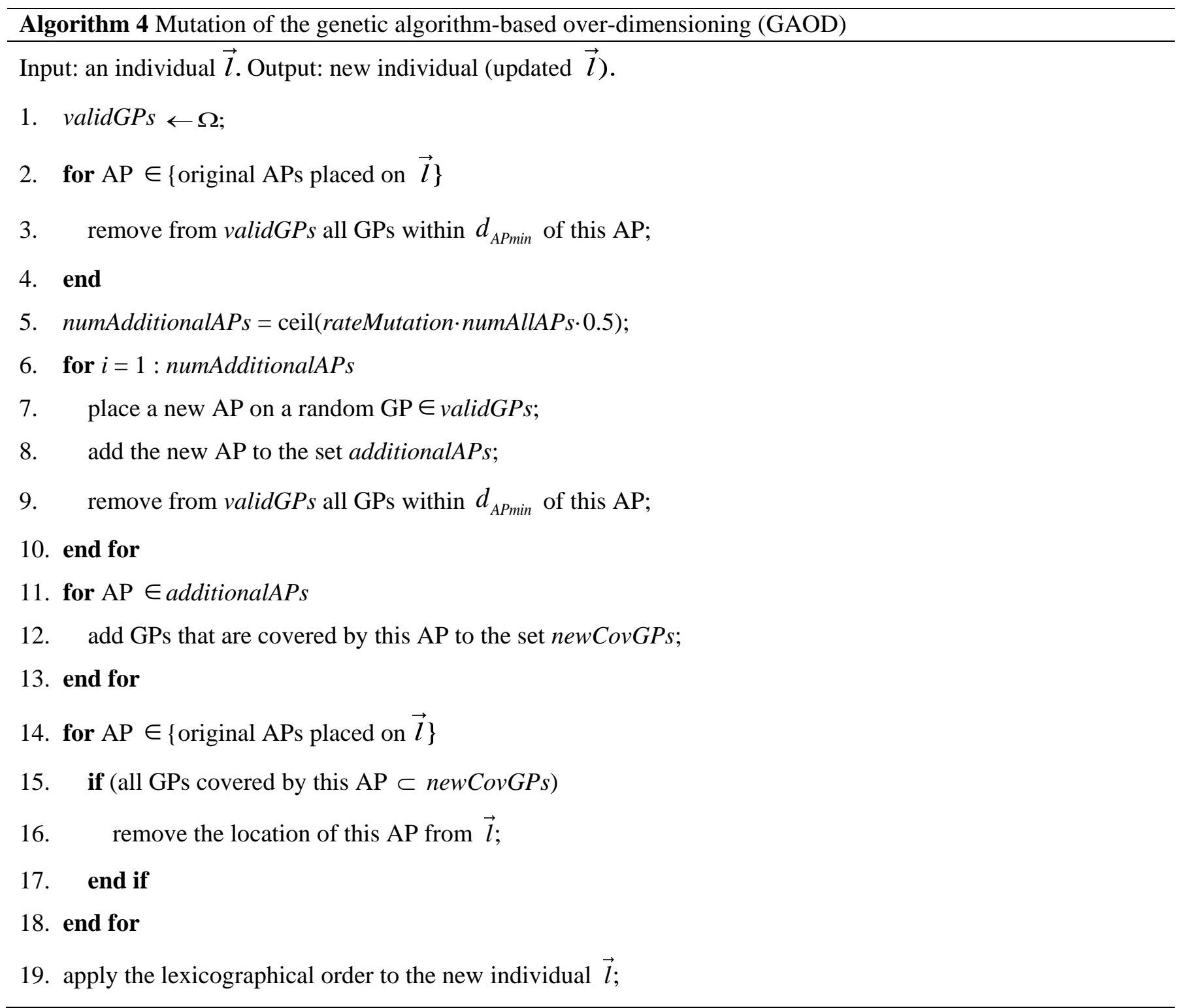

AP separation. At step 2 (lines 11-18), each AP in the original OD solution is checked whether it can be removed, while still satisfying the constraint of full double coverage defined by Eq. (5). The final new solution is reordered by following the lexicographical order (line 19).

Analogously, for memory efficiency, store memory-consuming variables (such as validGPs in Algorithm 4) are not locally stored in each individual. Instead, they are locally generated. The huge memory taken by these variables will consequently be freed up at the end of Algorithm 4.

\subsection{Parallel genetic algorithm}

A GA exhibits an intrinsic characteristic of parallelism, because it does not evaluate and improve a single solution but analyzes and modifies a set of solutions simultaneously (Wang et al., 2009). Therefore, instead of being viewed as a mono-thread algorithm, it can be seen as a "divide and conquer" algorithm, also referring to as "map and reduce". 


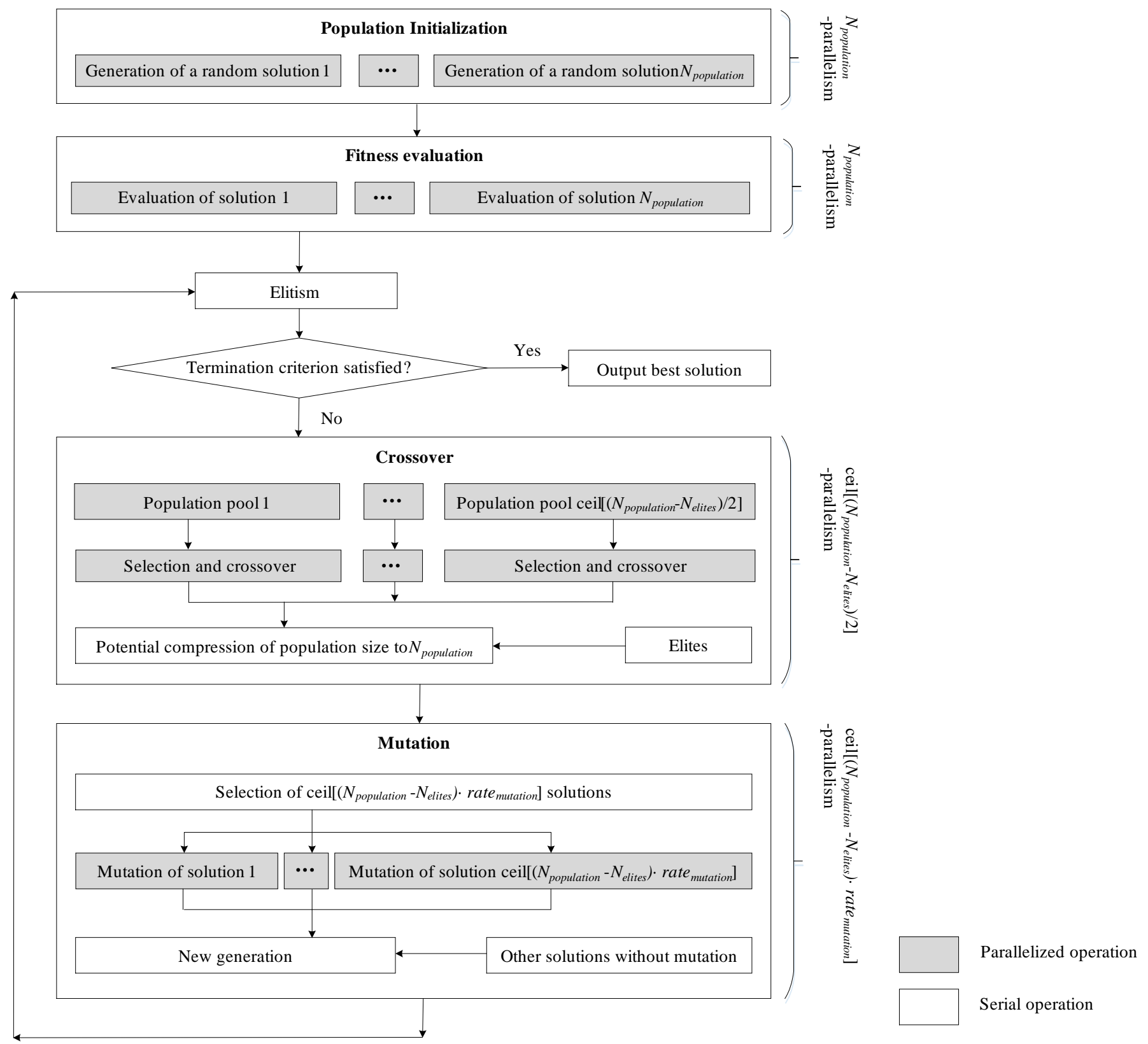

Fig. 7. Flowchart of the proposed parallel genetic algorithm

As to the "map" phase, the data space is split into smaller and independent chunks to be processed. Once the chunks are processed, partial results are collected to form up the final result, which is the "reduce" phase.

Accordingly, parallel computing (such as multithreading) (Hwu, 2014) can be used to shorten the runtime of largescale optimization. The substructures of a classical GA where the parallel computing can be applied include initial population generation, crossover and mutation of two individuals, and fitness calculation of a generation.

Fig. 7 presents the structure of the parallel GA for solving a large-scale OD problem. The number of parallelism depends on each genetic operator. In population initialization, the random generation of $N_{\text {population }}$ individuals is completely standalone, such that $N_{\text {population }}$ instances of Algorithm 2 can be parallelized. Similarly, $N_{\text {population }}$ fitness evaluations are parallelized at the end of a generation. To unlock the parallelism potential of crossover and mutation, 
the two operators are performed based on a population, instead of two individuals and one individual, respectively, in a decent GA. For a generation, the numbers of parallel crossover and mutation operations are ceil[ $\left.\left(N_{\text {population }}-N_{\text {elites }}\right) / 2\right]$ and ceil $\left[\left(N_{\text {population }}-N_{\text {elites }}\right) \cdot\right.$ rate $\left._{\text {mutation }}\right]$, respectively.

\subsection{Additional speedup measures}

As described in the former subsections, the design of ODGA follows the idea of enhancing search efficiency and reducing the memory and the computation time as much as possible, to facilitate large-scale optimization. Next to this, additional specific speedup measures are taken on two types of calculations which are extensively employed in ODGA.

First, $d_{\max }$ is calculated by the path loss model in advance and stored as a constant, instead of repeating the same path loss calculation for millions of times during a genetic search. Second, Algorithms 2-4 extensively search the area that an AP can cover with $T P_{\max }$, as well as the area which is within $d_{A P \min }$ of an AP. Instead of a rude iteration of all GPs in the environment to find the qualified GPs, such a search is only restricted within the $d_{\text {max }} \times d_{\text {nax }}$ and $d_{\text {APmin }} \times$ $d_{A P \min }$ rectangular areas which are centered at the investigated AP.

\section{Experimental validation}

The proposed GAOD was experimentally validated in a small open environment $(\approx 10 \mathrm{~m} \times 10 \mathrm{~m})$ in the factory hall of an automated guided vehicle (AGV) manufacturer, in Flanders, Belgium.

\subsection{Facilities, configurations, and measurements}

The WLAN coverage measurement facilities include (1) 4 Siemens ${ }^{\circledR}$ industrial APs (Scalance W788-2) with individual power supply, (2) a Zotac ${ }^{\circledR}$ mini-PC as a wireless client that receives signals from the APs, (3) an AGV as

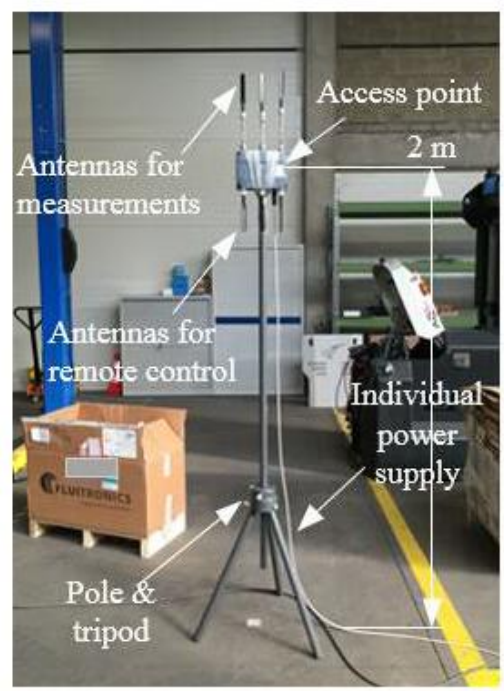

(a) Transmiter: one of the four access points deployed in the environment

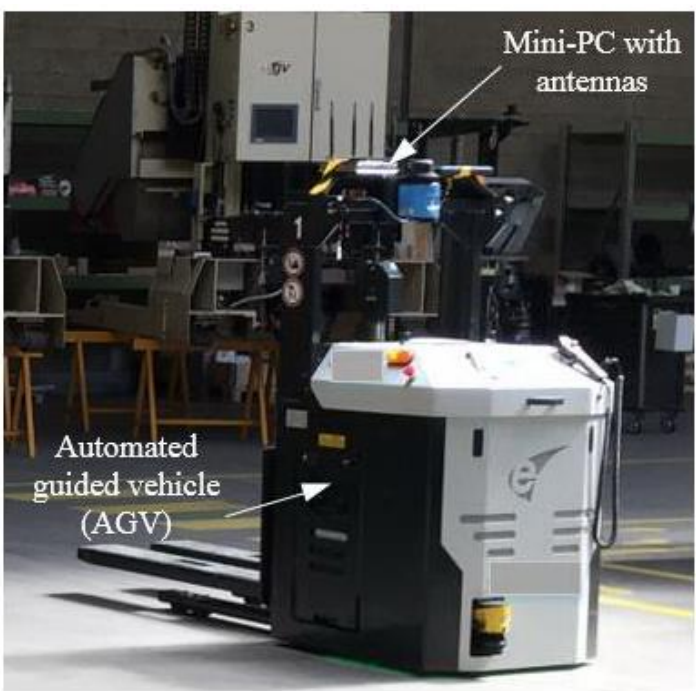

(b) Receiver: mobile client composed of an automated guided vehicle and a mini-PC with antennas

Fig. 8. Wireless signal transmitter and receiver for empirical measurements 
the controllable mobile vehicle which carries the client on the top such that the shadowing effects of AGV itself is prevented, (4) 4 poles with tripods to mount the APs, and (5) a central software system that controls measurements in terms of AGV location recording, synchronization with the measurement database in the mini-PC, and design of measurement experiments, e.g., assignment of wireless signal metrics to be measured and duration of an experiment. While the fifth measurement facility was a laptop, Fig. 8 demonstrates the first four measurement facilities which are grouped to the transmitter and receiver sides.

As placing APs within the environment would impede the AGV's movement, the 4 APs were constrained to be placed only on the four boundaries of the environment. The minimal AP separation distance $d_{A P \min }$ was set as $5 \mathrm{~m}$. Besides, 44- $\mathrm{dB}$ attenuators were installed to all transmit radio interfaces of the four APs, so as to mimic a larger environment that needs four APs for double full coverage. The proposed GAOD was implemented in the central measurement control system to provide an OD solution for this environment. Table 3 further lists the configurations for APs, the mobile client, and the GAOD.

Table 3

\section{Configurations of the environment and genetic algorithm based over-dimensioning (GAOD)}

\begin{tabular}{ll}
\hline & Wireless configurations \\
\hline Shadowing margin (95\%) & $1 \mathrm{~dB}$ \\
Fading margin (99\%) & $0 \mathrm{~dB}$ \\
Interference margin & $0 \mathrm{~dB}$ \\
AP antenna attenuation & $44 \mathrm{~dB}$ \\
WLAN standard & IEEE 802.11n \\
Frequency band & $2.4 \mathrm{GHz}$ \\
AP height & $2 \mathrm{~m}$ \\
AP only on the wall? & Yes \\
Minimal AP separation ( $d_{A P \text { min }}$ ) & $5 \mathrm{~m}$ \\
Client height & $1.8 \mathrm{~m}$ \\
Required physical bitrate of a client & $24 \mathrm{Mbps}$ \\
Required minimal sensitivity of a client & $-79 \mathrm{dBm}$ \\
\hline & Genetic algorithm configurations \\
\hline Population size & 100 \\
Elitism rate & $10 \%$ \\
Crossover rate & $90 \%$ \\
Mutation rate & $5 \%$ \\
Maximum iteration & 30 \\
\hline
\end{tabular}



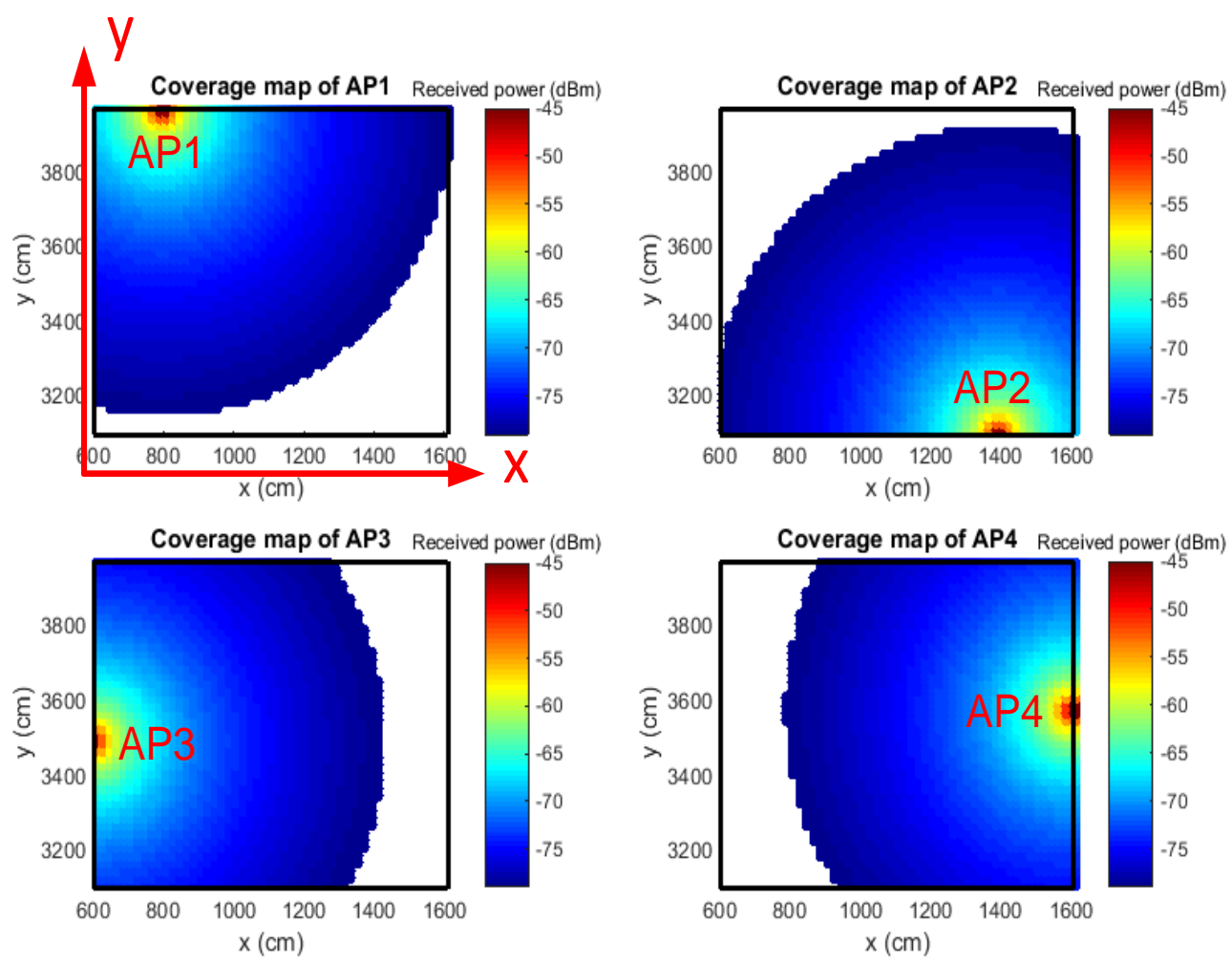

Fig. 9. Coverage of the four over-dimensioned access points (APs), which is predicted by the path loss model. The colored area is covered by an AP, while the white area is out of coverage.

Upon the start of an experiment, the measurement task was remotely sent from the measurement control system to the mini-PC-based client. This client then connected to the specified AP and started to record the received signal strength and the corresponding time for each record. During an experiment, the AGV was controlled to drive around the environment at $20 \mathrm{~cm} / \mathrm{s}$ such that the AGV path swept the environment as evenly as possible. Meanwhile, the measurement control system recorded the real-time AGV 2D locations by communicating to the AGV backend server. At the end of an experiment, the AGV and the client stopped moving and measuring the received signal strength, respectively; the measurement control system retrieved the measured RSSI from the client and attached a 2D location to each sample by synchronization. Such an experiment was used for two objectives: (1) characterization of radio propagation in the environment which determines the variables of the one-loss path loss model, i.e., Eq. (3), and leads to an empirical OD solution, (2) monitoring of the wireless coverage when the 4 APs are deployed according to the empirical OD solution.

\subsection{Measurement results}

To build an empirical path loss model, $3745 \mathrm{RF}$ power samples were collected. Regression was applied to these data to build an empirical path loss model (Tanghe et al., 2008) formulated by Eq. (3). Consequently, PL0 was 39.87 and $n$ was 1.78 . The $\mathrm{R}$-squared value was $97.38 \%$, indicating a high fitness level of this empirical path loss model, compared to the measured data. The GAOD then used this empirical path loss model to produce an OD solution. The 

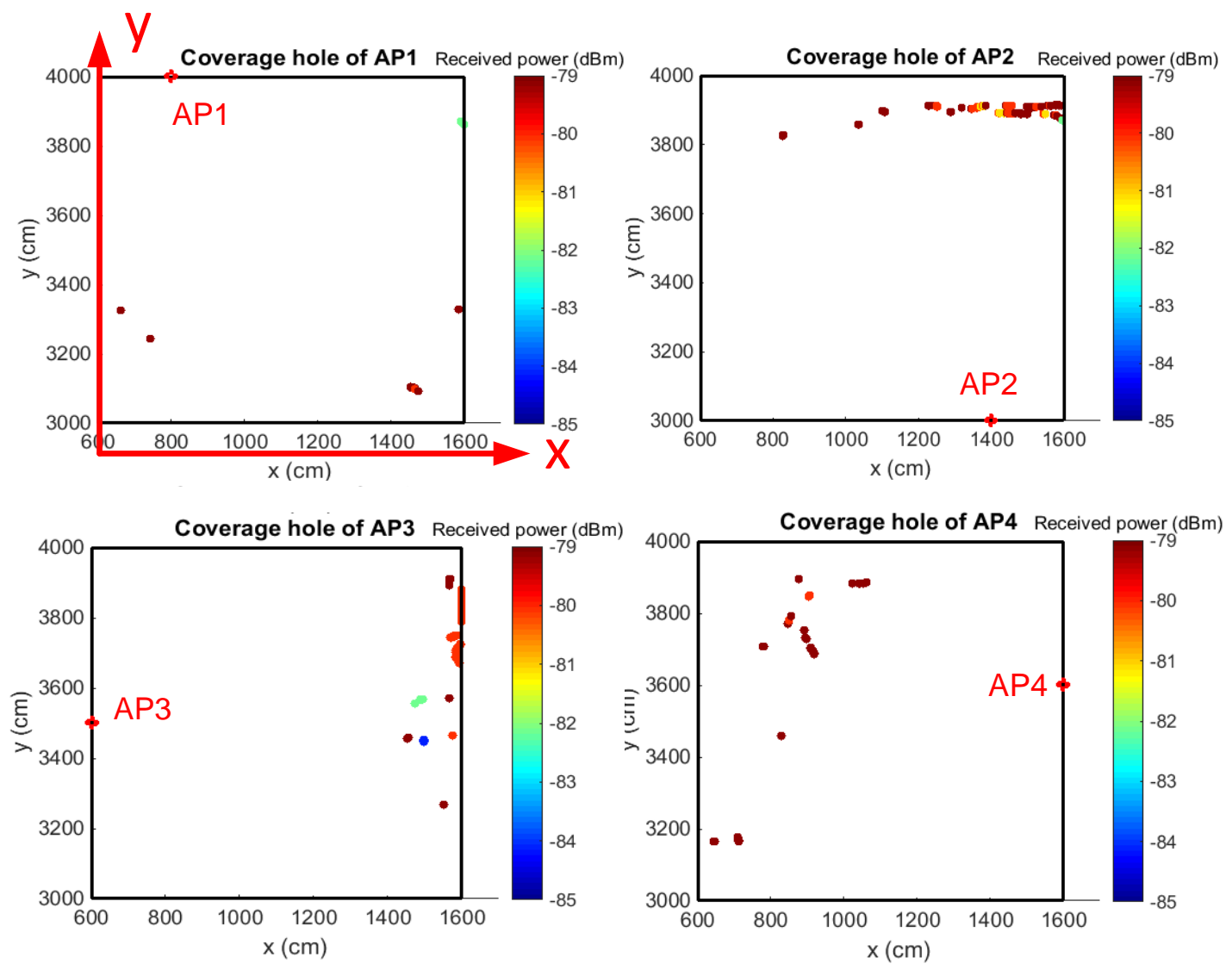

Fig. 10. Coverage hole of the four over-dimensioned access points (APs), which is measured by experiments.

obtained OD solution is illustrated in Fig. 9. The thick black lines represent the four boundaries of the environment. The 4 APs are placed on the boundary of the environment, such that two full coverage layers are envisioned to be guaranteed. Each AP has the exact 2D location (in $\mathrm{cm}$ ), as depicted in Fig. 9.

Fig. 9 also exhibits the coverage of each over-dimensioned AP. The red represents the area with high RF power, the blue represents the area with low RF power which is whereas not lower than the required minimal sensitivity $(-79 \mathrm{dBm}$, Table 3), while the white stands for the area that cannot be covered by the AP. As shown, every AP cannot fully cover the environment. However, two APs can form up a complete coverage layer by combing the respective coverage, i.e., AP1 and AP2, as well as AP3 and AP4. The minimal inter-AP distance (5.13 m) is the one between AP1 and AP3. This is larger than the preset $d_{A P \text { min }}(5 \mathrm{~m})$. Therefore, this numerically demonstrates the effectiveness of the ODGA.

The 4 APs were then placed in the environment according to the obtained OD solution. The coverage of each AP was measured by following the experiment procedure described in Sect. 6.1. As a result, Fig. 10 presents the received power samples that are lower than the required minimal sensitivity, i.e., coverage hole of each AP. The coverage hole of an AP is always close to the environment boundary and on the opposite side of this AP location. The coverage power samples vary between $-79 \mathrm{dBm}$ and $-85 \mathrm{dBm}$, which are actually below the present sensitivity $(-79 \mathrm{dBm})$. Therefore, this empirically demonstrates the effectiveness of the proposed ODGA. 


\section{Numerical experiments}

The focus of numerical experiments is on the algorithmic scalability beyond the experimental validation at a small scale (Sect. 6), to adapt to the real industrial wireless deployment scale. The models and algorithms were implemented in Java. The numerical experiments were performed on a PC running 64-bit Win7 and with an Intel i5-3470 CPU (two 3.20 GHz single-thread cores) and an 8 GB RAM.

\subsection{Configurations}

The two industrial indoor environments under investigation are, respectively, a factory hall of an automated guided vehicle (AGV) manufacturer and a warehouse of a car manufacturer, both located in Flanders, Belgium.

The factory hall measures $102 \mathrm{~m} \times 24 \mathrm{~m}$. Metal racks are placed inside for component storage. AGVs of varying sizes are placed usually without moving and waiting for integration, maintenance, or shipment. Wide WiFi coverage is needed for AGV communication and Internet access of onsite laptops.

The warehouse measures $415 \mathrm{~m} \times 200 \mathrm{~m}$. Metal racks are placed inside, at a height of nine meters. Wooden boxes that contains metal components are placed on the racks. Wide WiFi coverage is required to support voice picking. The pickers are equipped with microphones and earphones. They communicate with the control center via WLANs to pick up and place a stuff at a specific location.

Mapping to the OD model, a metal rack in both cases is an obstacle that potentially causes evident shadowing effects to radio propagation. In the following experiments, an obstacle measures $20 \mathrm{~m} \times 3 \mathrm{~m} \times 9 \mathrm{~m}$. It can be placed either horizontally (the length side is parallel to the length side of the environment) or vertically (the length side is parallel to the width side of the environment). The direction and location of an obstacle are randomly and uniformly generated in the environment. The number of racks is an input of the OD model. The GPs that are taken up by obstacles are not considered for the path loss calculation.

The experiment-related parameters are shown in Table 4, including the path loss model, the AP transmitter, the receiver, the environment and the GAOD. All APs are powered on with maximal transmit power $\left(T P_{\max }\right)$. The grid size $(g s)$ is set as one meter. It is within the distance of 10 wave length $(\approx 1.2 \mathrm{~m})$ at $2.4 \mathrm{GHz}$ radio frequency band, meaning that the path loss within this distance can be considered constant without sacrificing the precision of path loss calculation. The two parameters $P L 0$ and $n$ for the one-slope path loss model were the same as these in Sect. 6. The path loss caused by a metal rack $(7.37 \mathrm{~dB})$ was the mean value of the measured path loss data.

Furthermore, two benchmark algorithms are used to evaluate the performance of GAOD: the GHOD and the random placement (Algorithm 2). Though the latter looks naif compared to a genetic algorithm, it is a common method to deploy wireless sensors (Jain and Ramana Reddy, 2015). 


\subsection{Results in a small-scale empty environment}

The GHOD and the GAOD were first performed in the small-scale empty environment (i.e., factory hall of the AGV manufacturer), by loosening the constraint in Eq. (7) such that no metal obstacles exist (i.e., $N_{o}=0$ ). The performance metrics of both algorithms are shown in Table 5. Both algorithms satisfy the constraints of two full coverage layers

\section{Table 4}

\section{Numerical experiment configurations}

\begin{tabular}{|c|c|}
\hline \multicolumn{2}{|r|}{ Path loss model } \\
\hline PL0 & $39.87 \mathrm{~dB}$ \\
\hline$n$ & 1.78 \\
\hline Shadowing margin (95\%) & $1 \mathrm{~dB}$ \\
\hline Fading margin $(99 \%)$ & $0 \mathrm{~dB}$ \\
\hline Interference margin & $0 \mathrm{~dB}$ \\
\hline \multicolumn{2}{|r|}{ An access point (AP) as the transmitter } \\
\hline Height & $2 \mathrm{~m}$ \\
\hline Gain & $3 \mathrm{~dB}$ \\
\hline WiFi standard & IEEE $802.11 n$ \\
\hline Frequency band & $2.4 \mathrm{GHz}$ \\
\hline Maximal transmit power & $7 \mathrm{dBm}$ \\
\hline Only on the wall? & No \\
\hline \multicolumn{2}{|r|}{ Receiver of a wireless client } \\
\hline Height & $1.4 \mathrm{~m}$ \\
\hline Gain & $2.15 \mathrm{~dB}$ \\
\hline Required physical bitrate & $54 \mathrm{Mbps}$ \\
\hline Required minimal sensitivity & $-68 \mathrm{dBm}$ \\
\hline \multicolumn{2}{|r|}{ Environment } \\
\hline Size of the factory hall (small) & $102 \times 24 \mathrm{~m}(2600$ grid points $)$ \\
\hline Size of the warehouse (large) & $415 \mathrm{~m} \times 200 \mathrm{~m}$ (83616 grid points) \\
\hline Grid size $(g s)$ & $1 \mathrm{~m}$ \\
\hline Frequency band & $2.4 \mathrm{GHz}$ \\
\hline Antenna type & Omnidirectional \\
\hline Minimal inter-AP separation & $5 \mathrm{~m}$ \\
\hline Metal rack size & $20 \mathrm{~m} \times 3 \mathrm{~m} \times 9 \mathrm{~m}$ \\
\hline Path loss caused by one metal rack & $7.37 \mathrm{~dB}$ \\
\hline
\end{tabular}




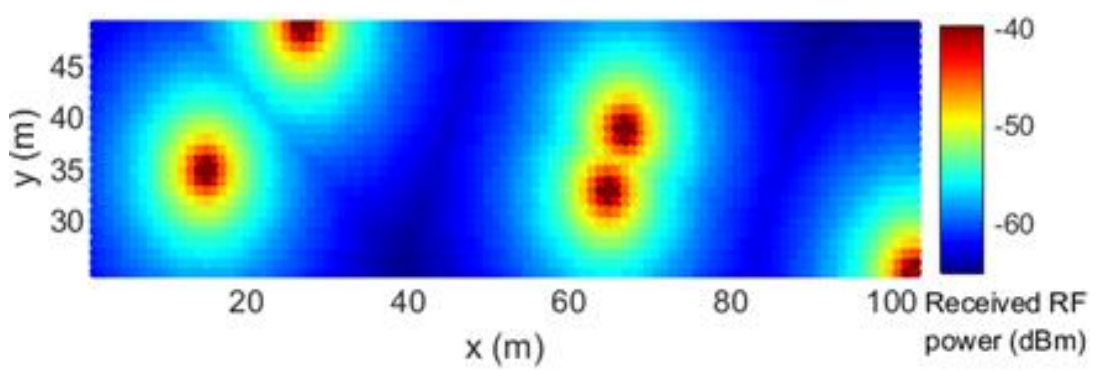

(a) Solution of GHOD

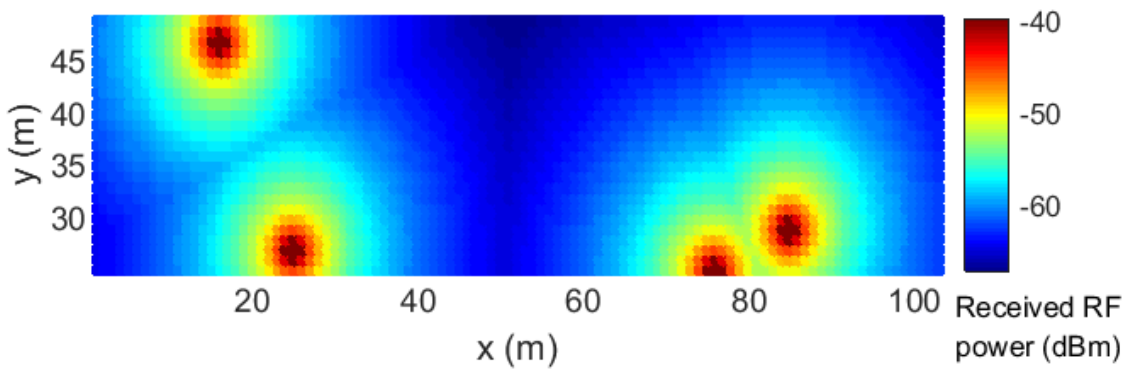

(b) Solution of GAOD

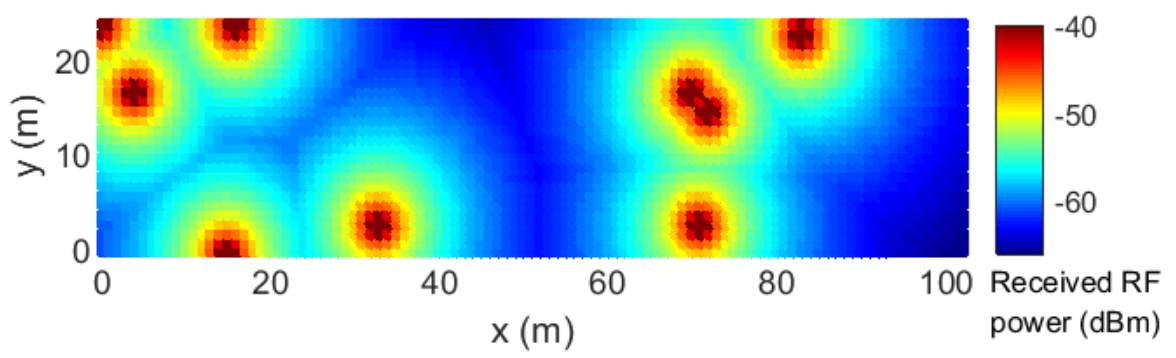

(c) Random OD solution

Fig. 11. Over-dimensioning solution comparison in a small-scale empty environment.

Population size

Elitism rate

Crossover rate

Mutation rate

Stop criterion
30 (small-scale environment), 100 (large-scale environment)

$$
8 \%
$$

$95 \%$

$5 \%$

No improvement of the best fitness value during 10 consecutive cycles

and minimal AP separation $\left(d_{A P \min }\right)$ in the target factory hall. However, the GAOD outputs one AP less to solve the same OD model, and is 2.7 times faster than the GHOD.

In the solution output by the GHOD, the number of GPs that are covered by at least three APs is 3.3 times as the same type of number in the GAOD. This unveils an intrinsic characteristic of GAOD: it essentially minimizes the number of GPs that are covered by more than two APs, while ensuring each GP is covered by at least two APs.

Moreover, 230 random OD solutions are generated by using Algorithm 2. As indicated in Table 5, on average five APs are needed with a standard deviation of one AP. This means that the median case corresponds to the GHOD, and the worst case outputs 50\% more APs than GAOD, which accordingly leads to about 50\% more AP deployment cost than GAOD. The time to generate a random solution is negligible. This is normal since a random instance only needs 
to conform to the two fundamental constraints (double full coverage and minimal AP separation) defined by Eqs. $(5,11)$, without any optimization effort. Overall, both proposed algorithms can give effective OD solutions, while GAOD is superior to GHOD on the small scale in terms of AP number that is output and computation time.

As a comparison, the OD solutions output by the GHOD, the GAOD and the random OD (Algorithm 2) are shown in Fig. 11. The $\mathrm{x}$ and $\mathrm{y}$ axes are the horizontal and vertical sides of the factory hall under investigation, respectively. The highest received power of each GP is visualized. High power is represented by red, while low power is indicated by blue. As a result, the over-dimensioned APs are represented by red dots. Fig. 11 evidently shows that the GAOD outputs the least APs, while the random generation outputs the most APs within the same environment. In the solution of GAOD, APs tend to be evenly distributed over the environment. In the solution of random generation, APs however tend to be clustered, which also reveals why more APs are needed for satisfying the same constraints of the same OD model.

Fig. 11 also serves as a heat map for network managers and plan managers. It vividly reveals the coverage of the whole industrial indoor environment. The minimal received power on this map is $-67 \mathrm{dBm}$, which is higher than the

threshold $-68 \mathrm{dBm}$ (Table 4). The minimal inter-AP separation is $9.8 \mathrm{~m}$, which is larger than $d_{A P \min }(5 \mathrm{~m})$ constrained by Eq. (11).

\subsection{Results in a large-scale empty environment}

The GHOD and the GAOD were then performed in the large-scale empty environment (i.e., warehouse of the car manufacturer), by making loose the constraint in Eq. (7) such that no metal obstacles exist (i.e., $N_{O}=0$ ). The performance metrics of are presented in Table 5. Both algorithms meet with the two essential constraints, i.e., double full coverage, and any AP beyond $d_{A P \min }$ of all the other APs. Most importantly, in terms of the optimization objective, the GAOD outputs six APs less. This roughly corresponds to 7.4\% reduction of the network deployment cost.

The GHOD is 7.5 times faster than the GAOD. This is inverse to the phenomenon revealed in the former case. It is explained by the $O(n)$ time complexity of GHOD, of which the fast performance shows up when the problem size grows rapidly. Nevertheless, the time taken is not a crucial factor for wireless planning, since the planning is performed only once or at a very low frequency. Besides, the time $(5.5 \mathrm{~h})$ taken by the GAOD is considered acceptable, and is significantly improved compared to $173.6 \mathrm{~h}$ in the former experiment of running the $\mathrm{d}_{\mathrm{avg}}$ criterion (Sect. 4).

In the OD solution given by the GHOD, 9\% more GPs are covered by at least three APs. This again demonstrates the aforementioned GAOD's intrinsic characteristic of global optimization. 


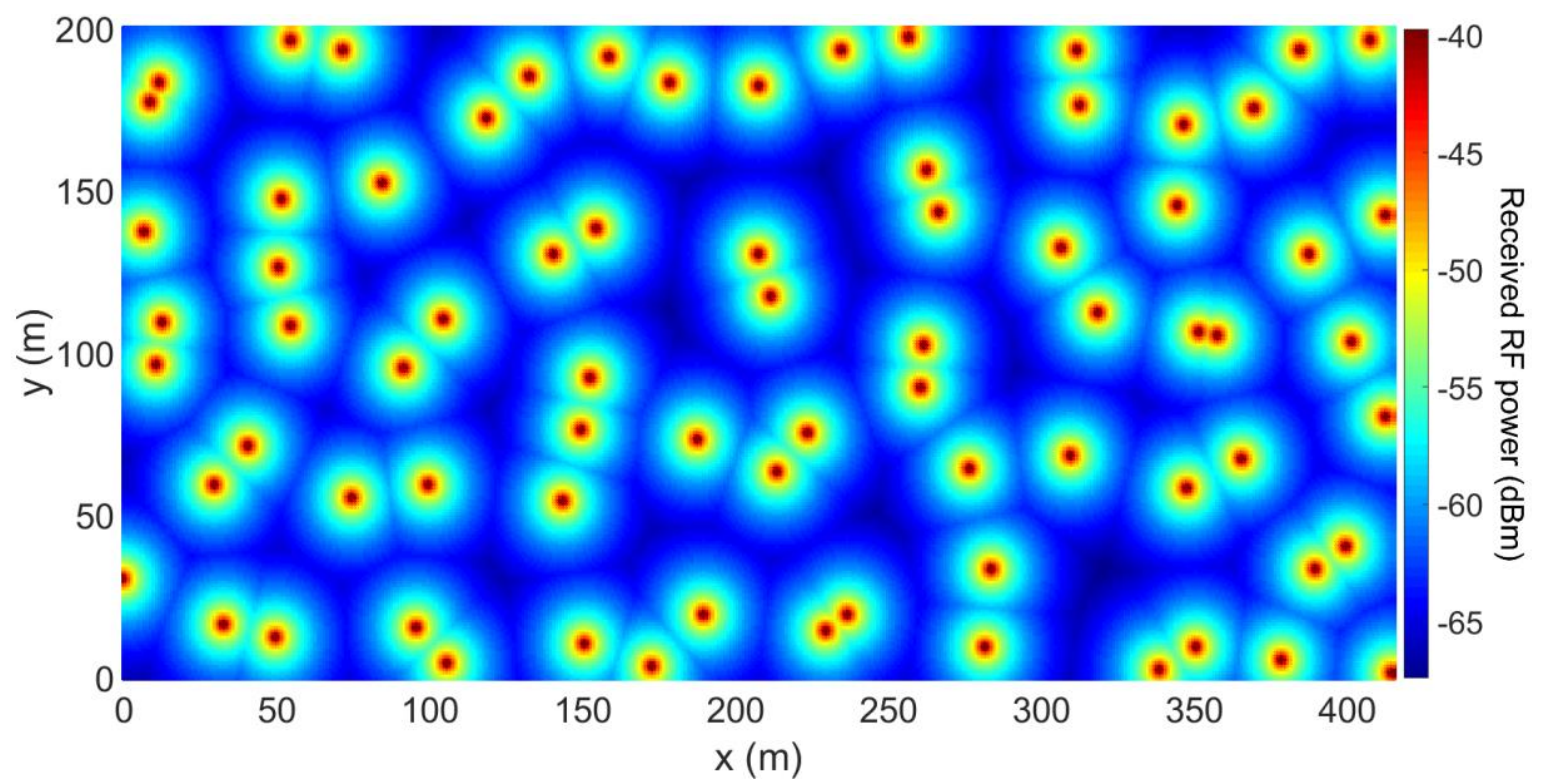

Fig. 12. Over-dimensioning solution given by the GAOD in a large-scale empty environment.

Furthermore, 380 random solutions are generated. As shown in Table 5, 85 APs in average are needed with a standard deviation of three APs. This mean AP number is $4.9 \%$ and $13.3 \%$ larger than the number of APs output by the GHOD and the GAOD, respectively. The best case in the random OD solution has 82 APs, which is still worse than the performance of GHOD and GAOD, due to the increasing AP deployment cost. The time for establishing an OD solution is much shorter than the GHOD and the GAOD, which is the same phenomenon as in the small scale due to the same reason. The percentage of GPs that are covered for more than twice is higher than the GAOD, and is at the similar level of GHOD.

The OD solution given by the GAOD is further visualized in Fig. 12. The minimal received power on this heat map is $-67.42 \mathrm{dBm}$, which is higher than the threshold. The minimal inter-AP separation among all the over-dimensioned APs is $6 \mathrm{~m}$, which is higher than $d_{A P \min }(5 \mathrm{~m})$.

Table 5

Algorithm performance in empty environments

\begin{tabular}{|c|c|c|c|c|c|c|}
\hline \multirow{2}{*}{ Performance metric } & \multicolumn{2}{|c|}{ GHOD } & \multicolumn{2}{|c|}{ GAOD } & \multicolumn{2}{|c|}{ Random OD (mean/deviation) } \\
\hline & $\mathrm{S}^{\mathrm{a}}$ & $\mathrm{L}^{\mathrm{b}}$ & $\mathrm{S}$ & $\mathrm{L}$ & $\mathrm{S}$ & $\mathrm{L}$ \\
\hline Number of all APs & 5 & 81 & 4 & 75 & $5 / 1$ & $85 / 3$ \\
\hline Runtime (sec) & 8 & 2633 & 3 & 19789 & $0 / 0$ & $522 / 142$ \\
\hline Percentage of GPs covered at least twice & 100 & 100 & 100 & 100 & $100 / 0$ & $100 / 0$ \\
\hline Any AP separation within $d_{A P \min }$ ? & No & No & No & No & No & No \\
\hline Percentage of GPs covered more than twice & 70 & 93 & 21 & 84 & $65 / 18$ & $91 / 2$ \\
\hline
\end{tabular}

${ }^{a}$ Small-scale environment. ${ }^{b}$ Large-scale environment. 


\subsection{Results in obstructed environments}

The GHOD and GAOD algorithms were further performed in the small-scale and large-scale obstructed environments, respectively. One and ten metal racks (Table 4) were randomly placed in the small-scale and large-scale environments, respectively. In total, 330 and 200 random OD solutions were generated in the small-scale and largescale environment, respectively.

All the obtained OD solutions meet with all the constraints of the model. Table 6 lists the other key performance metrics for comparison. All the solutions are obtained within a reasonable time span, regarding the problem size and context of wireless planning. However, the GAOD can output the least APs in both small and large environments that have metal racks. A random solution outputs $40 \%$ more APs in average and $60 \%$ more APs in the worst case, compared with the GAOD. The number of APs that are over-dimensioned by the GHOD is intermediate, compared with the other two algorithms.

Moreover, the percentage of GPs that are covered more than twice exhibits a similar performance trend: the GAOD achieves the lowest percentage, while a random solution and the GAOD have an evidently higher percentage. In a small-scale obstructed environment, the percentage of GPs that are covered at least twice is $22 \%$ less in GAOD than that in GHOD, and is in average $9 \%$ less than that of a random solution. In a large-scale obstructed environment, the percentage of GAOD is $6 \%$ less than that of GHOD, and is in average 5\% less than that of a random solution. The difference between the GAOD and the other two algorithms reduces in a large-scale environment. 
Table 6

Algorithm performance in obstructed environments

\begin{tabular}{llccc}
\hline Algorithm & & Number of APs & Runtime (sec) & \% of GPs covered at least twice \\
\hline \multirow{2}{*}{ GHOD } & $\mathrm{S}^{\mathrm{a}}$ & 6 & 2 & 88 \\
& $\mathrm{~L}^{\mathrm{b}}$ & 91 & 23956 & 91 \\
\hline \multirow{2}{*}{ GAOD } & $\mathrm{S}$ & $\mathbf{5}$ & 7 & $\mathbf{6 6}$ \\
& $\mathrm{L}$ & $\mathbf{8 3}$ & 34028 & $\mathbf{8 5}$ \\
\hline \multirow{2}{*}{ Random OD (mean/deviation) } & $\mathrm{S}$ & $7 / 1$ & $0 / 0$ & $75 / 11$ \\
& $\mathrm{~L}$ & $92 / 2$ & $543 / 54$ & $90 / 1$ \\
\hline
\end{tabular}

${ }^{\mathrm{a}}$ Small-scale environment. ${ }^{\mathrm{b}}$ Large-scale environment.

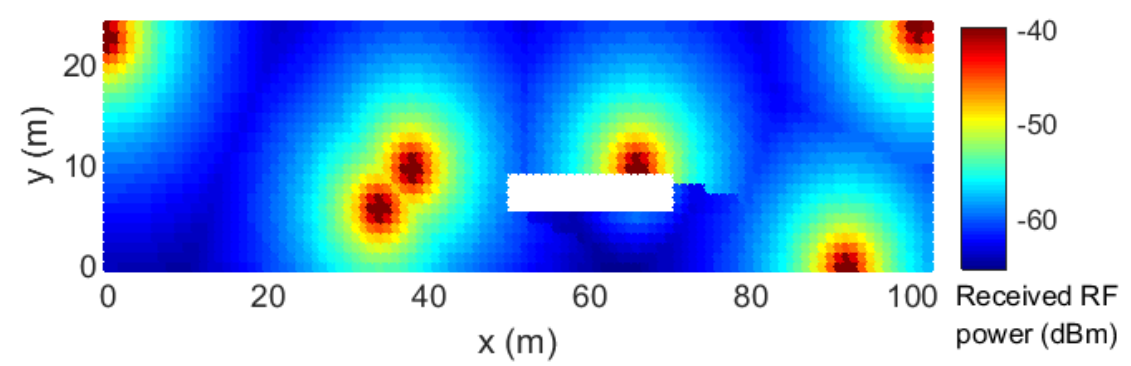

(a) Solution of GHOD

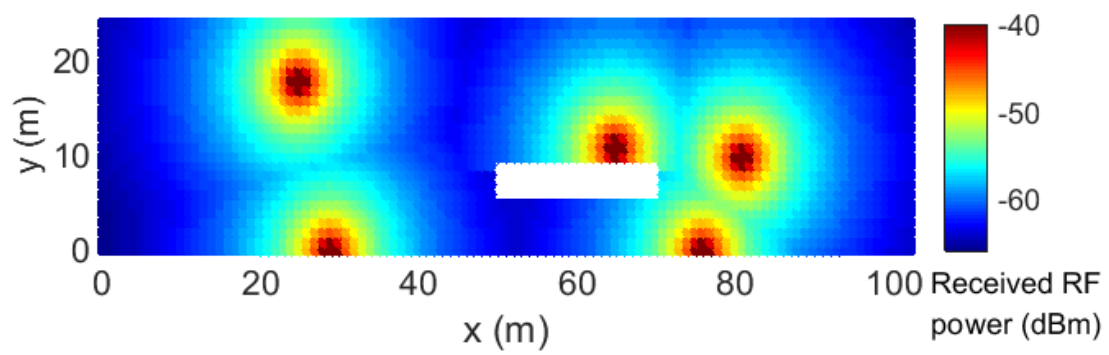

(b) Solution of GAOD

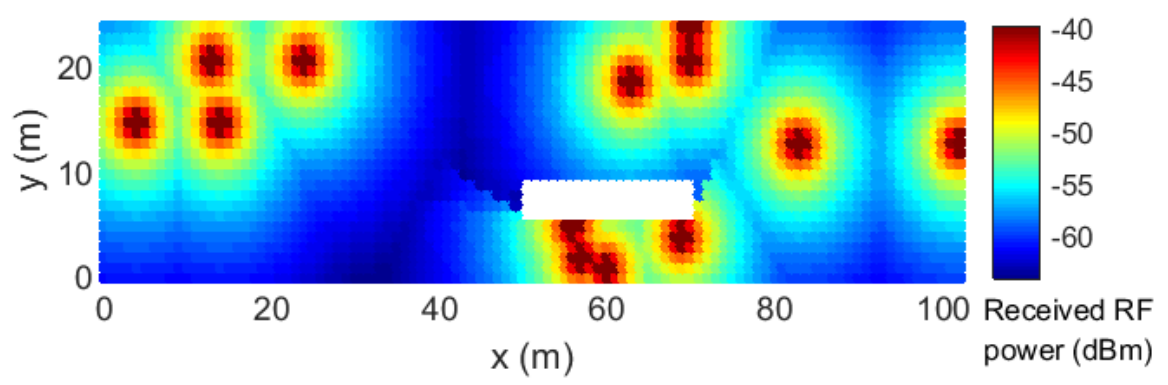

(c) Random OD solution

Fig. 13. Over-dimensioning solution comparison in a small-scale obstructed environment (the white rectangle represents a metal rack). 


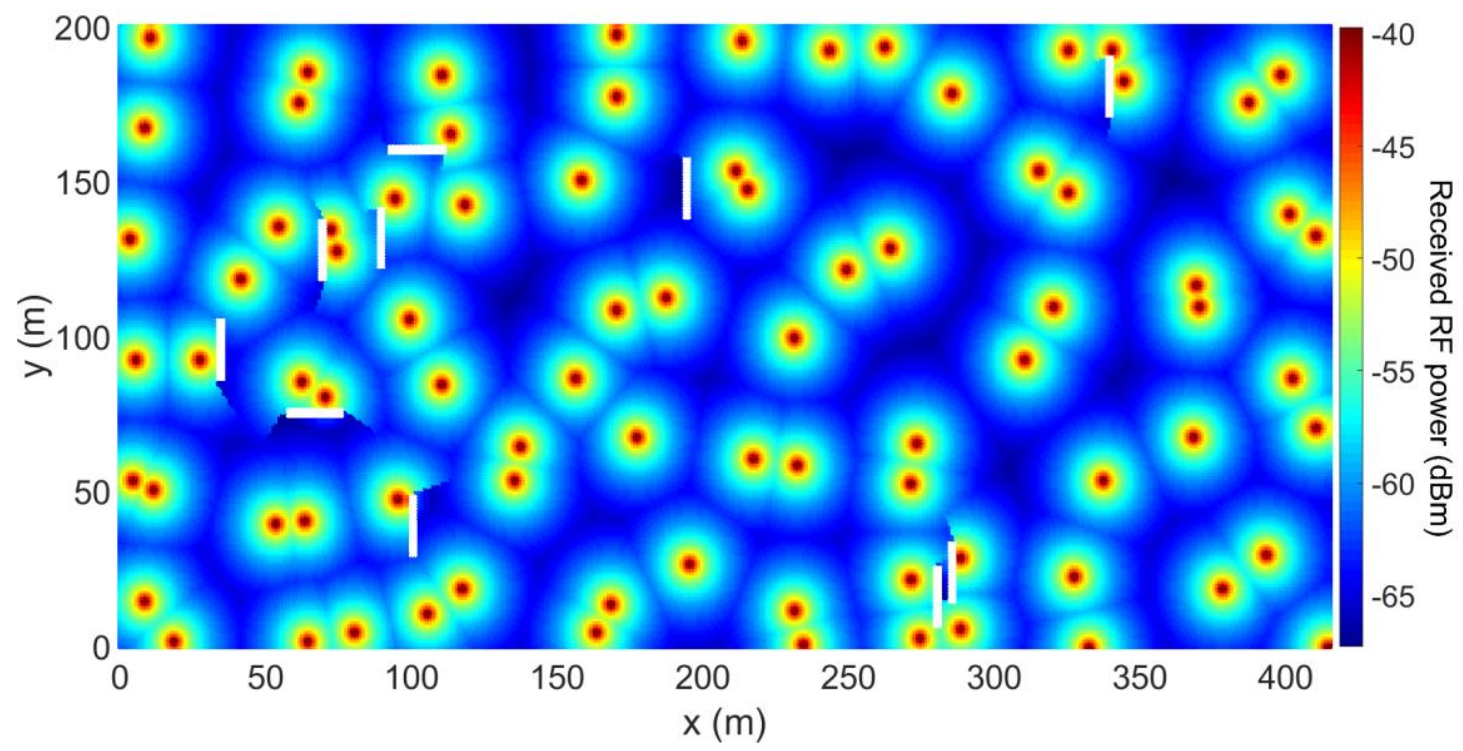

Fig. 14. Over-dimensioning solution given by the GAOD in a large-scale obstructed environment (the 10 white rectangles represent 10 metal racks).

One important reason for this phenomenon is that more metal racks are placed in the large-scale environment, which causes more shadowing effects. Consequently, more additional APs are needed to specifically tackle these shadowing effects, while increasing the coverage layer number of the other GPs that are already covered twice.

A performance comparison is made between the OD solutions, which are output by the same algorithm in the same environment without and with metal, respectively. In the small-scale environment, more APs are output by the three algorithms if dominant metal is present. The AP number increasing rate of a random solution is the highest, at $40 \%$ in average. This is explained by a lack of effective optimization measures in random solution generation. In reverse, this demonstrates the effectiveness of GHOD and GAOD, in terms of minimizing the AP number. In the large-scale environment, similarly, more APs are needed under the presence of dominant metal. The AP number increasing rate of the three algorithms is on the same level around 10\%, while the rate of GHOD is the highest, at $12 \%$. This shows that the optimization performance of GHOD is worse than that of GAOD.

The coverage map of the OD solution in the small-scale obstructed environment output by the GHOD, the GAOD, and the random generation, is further visualized in Fig. 13. The superiority of GAOD is clearly demonstrated, in terms of minimizing the AP number while meeting with all the constraints of the OD model.

The coverage map of the OD solution in the large-scale obstructed environment output by the GAOD is further presented in Fig. 14. The 10 metals racks that are randomly generated are represented by 10 white rectangles. It is observed that APs tend to cluster around metal racks. This reveals the intrinsic property of GAOD when dealing with shadowing effects of dominant metal: additional APs are actually placed to tackle the additional path loss that is caused by these shadowing effects. 
Table 7

Deployment cost (number of APs $\times$ AP unit price) comparison of 6 different algorithms in obstructed environments (metal rack placement remains that in Figs. 13\&14)

\begin{tabular}{lcccc}
\hline Algorithm & \multicolumn{2}{c}{$\begin{array}{c}\text { Number of APs } \\
\text { (mean } \pm \text { standard deviation) }\end{array}$} & $\begin{array}{c}\text { Cost gap compared to the proposed GAOD } \\
\text { (positive: more expensive; negative: cheaper) }\end{array}$ \\
\cline { 2 - 5 } & $\begin{array}{c}\text { Small-scale } \\
\text { environment }\end{array}$ & $\begin{array}{c}\text { Large-scale } \\
\text { environment }\end{array}$ & $\begin{array}{c}\text { Small-scale } \\
\text { environment }\end{array}$ & $\begin{array}{c}\text { Large-scale } \\
\text { environment }\end{array}$ \\
\hline GHOD-1 $^{\mathrm{a}}$ & $3 \pm 0$ & $46 \pm 0$ & $-25 \%$ & $-39 \%$ \\
\hline GAOD-1 $^{\mathrm{b}}$ & $3 \pm 0$ & $44 \pm 1$ & $-25 \%$ & $-41 \%$ \\
\hline Random OD-1 & $4 \pm 1$ & $49 \pm 2$ & 0 & $-35 \%$ \\
\hline GHOD & $5 \pm 0$ & $81 \pm 0$ & $25 \%$ & $8 \%$ \\
\hline GAOD & $4 \pm 0$ & $75 \pm 1$ & 0 & 0 \\
\hline Random OD & $5 \pm 1$ & $85 \pm 3$ & $25 \%$ & $13 \%$ \\
\hline
\end{tabular}

${ }^{\mathrm{a}}$ Modified GHOD version that ensures only one full coverage layer.

${ }^{b}$ Modified GAOD version that ensures only one full coverage layer.

${ }^{c}$ Modified random OD version that ensures only one full coverage layer.

\subsection{Deployment cost analysis}

To analyze the deployment cost performance of the proposed GAOD, each of the following 6 algorithms went through 10 independent runs in small and large obstructed environments: GHOD-1, GAOD-1, random OD-1, GHOD, GAOD, and random OD. The former 3 algorithms are modified versions of the latter 3 algorithms, respectively, such that only one full coverage layer is ensured in a target environment, i.e., Eq. (5) is replaced by Eq. (14) while the objective and other constraints remain in the proposed OD model (Sect. 2.1).

$$
\sum_{j=1}^{N_{A P}} \alpha_{i j} \geq 1, \forall i \in I
$$

The number of APs is taken as the deployment cost indicator, as it is closely linked to the cost of deploying a homogeneous IWLAN. The cost gaps between deployment solutions output by different algorithms is thereby characterized by the gap between the numbers of APs.

As presented in Table 7, among the 6 algorithms, the most economical deployment solution is provided by the GAOD-1 (25\% and 41\% cheaper than the GAOD in small and large obstructed environments, respectively). As GAOD1 only guarantees 1 full coverage layer, the proposed GAOD is cost-effective in the sense that it at most requires an additional $41 \%$ cost to deploy an IWLAN that double cover a target environment. Among the 3 algorithms that establish two full coverage layers (GHOD, GAOD, and random OD), the GAOD also remains the most cost-effective, as is needs an $8 \%$ - 25\% cheaper deployment cost while ensuring double full coverage layers. Therefore, the proposed GAOD is able to provide factories cost-effective IWLAN deployment solutions.

Furthermore, the behavioral peculiarities of GHOD, GAOD and random OD are clearly exhibited in Table 7. The GHOD is deterministic with a constant number of APs (zero standard deviation) in different runs, while the GAOD and random OD are stochastic. An exception is observed in the GAOD, which also outputs a constant number of APs 
in the small obstructed environment. This could be explained by the small problem size, where the GAOD can rapidly converge toward the same optimal or nearly-optimal region in the same search space despite different runs.

\subsection{Discussion}

Several implications are further revealed based the former experiments. (1) As revealed by the comparison of Table 5 and Table 6, whether obstacles are considered will evidently influence the deployment cost of an IWLAN. It is also natural to reason that an increasing number of obstacles in the OD model will lead to more APs and thus a high deployment cost. Therefore, there should be a trade-off relationship between the robustness and deployment cost of an IWLAN. Although the layout of an industrial environment may alter causing a varying density of obstacles, it would be practical to consider a moderate density of obstacles when planning an IWLAN. Consequently, the robustness and cost performance of the deployed IWLAN can both achieve an acceptable level. (2) Despite the optimization efforts of the proposed GAOD in the deployment phase, the percentage of regions that are covered over twice is still significant, especially in a large industrial indoor environment (84\%, Table 5). This implies a high potential to further reduce the transmit power of deployed APs at the operational phase. In this way, the regions that are covered more than twice and the produced interference can be further reduced.

In addition to the demonstrated effectiveness and efficiency in deploying an economical robust IWLAN, the proposed GAOD is compared with the recent wireless network planning studies (Table 1). It has two major advantages. (1) The underlying OD model fills the gap in deploying a robust IWLAN. In contrast to the commonly used Boolean disk model, it explicitly investigates the 3D shadowing effects of dominant obstacles that are prevalent in an industrial indoor environment. These shadowing effects are integrated in an empirical one-slope path loss model, which enables simple yet accurate coverage prediction. Besides double full coverage layers, an AP spatial separation is ensured to avoid that APs covering the same region are simultaneously shadowed by an obstacle. (2) Compared to most recent studies that are limited within a small or medium problem size, the proposed GAOD can additionally perform largescale optimizations, where a large environment size, a higher spatial resolution, and a highly constrained deployment model are jointly involved. This complexity complies with the real requirement for deploying an IWLAN.

On the other hand, the proposed GAOD has two limitations (Table 1). (1) It focuses on homogeneous wireless network planning, while some recent studies investigate heterogeneous planning, e.g., planning of WLAN and LTE (Goudos et al., 2015). (2) The interference issue is not investigated. It would be of practical importance to limit or reduce interference in a dense wireless network. Furthermore, it needs dedicated measurement setups to empirically characterize a target environment, in order to deploy such a dense IWLAN by using the GAOD. Prior to empirical measurements, calibration should also be performed to ensure that the received power of a client is accurately sampled (Gong et al., 2016b).

\section{Conclusion}

Although wireless technologies are penetrating into the manufacturing industry, the existing research on wireless local area network (WLAN) planning is still mainly limited to small office environments. Consequently, the one 
coverage layer provided by these WLAN planning approaches is vulnerable to the shadowing effects of prevalent metal obstacles in harsh industrial indoor environments. To fill this gap, this paper investigates an over-dimensioning (OD) problem where two full coverage layers can be created at a large industrial scale for robust industrial wireless coverage. Although the second coverage layer serves as redundancy against shadowing, the deployment cost can be reduced by minimizing the number of access points (APs), while respecting the practical constraint of a minimal inter-AP spatial separation.

A genetic algorithm based OD (GAOD) algorithm is proposed to solve this problem. To enable large-scale industrial WLAN (IWLAN) planning, solution encoding, initial population generation, crossover and mutation are designed, such that the required computation time and memory are minimized. A greedy heuristic, named GHOD, is also proposed for benchmarking the performance of GAOD.

A factory hall $(102 \mathrm{~m} \times 24 \mathrm{~m})$ of an automated guided vehicle $(\mathrm{AGV})$ manufacturer and a warehouse $(415 \mathrm{~m} \times$ $200 \mathrm{~m}$ ) of a car manufacturer in Belgium are investigated as two case studies, i.e., small-scale and large-scale industrial indoor environment, respectively. Empirically, the feasibility and effectiveness of the OD model and GAOD is validated by measurements in a $10 \mathrm{~m} \times 10 \mathrm{~m}$ empty environment in the factory hall of the AGV manufacturer. Numerically, the effectiveness of GAOD and GHOD is extensively demonstrated in the two investigated environments, without and with the presence of metal racks, in comparison to the random OD solution generation. Compared to GAOD and GHOD, the random OD solution generation outputs up to $60 \%$ and $33 \%$ more APs, respectively. The superiority of GAOD, compared to GHOD, is demonstrated by the fact that GAOD outputs up to 20\% less APs for the same OD problem in a reasonable time span.

The outcome ODGA algorithm can help network managers and plant managers to automatically plan an IWLAN which has high availability under the presence of dominant metal in the environment. Moreover, it can easily be extended to plan other robust wireless networks such as wireless sensor networks.

This presented work can have fourfold future extensions. (1) A dedicated indicator may be introduced to quantify the robustness of deployed wireless networks in harsh industrial environments, regardless of the wireless network type. (2) The proposed OD model and GAOD algorithm can be extended from double to triple or even more coverage layers. This is especially important for wireless sensor networks, which may alternatively switch on/off each coverage layer to remain robust while conserving energy. (3) The interference of a dense network can be explicitly considered in the OD model as either a constraint or an objective for optimization. (4) Heterogeneous wireless networks can be simultaneously planned by considering other wireless technologies besides a WLAN.

\section{Acknowledgement}

This research was supported by the ICON-FORWARD project with imec.

\section{References}

Abdelkhalek, O., Krichen, S., Guitouni, A., 2015. A genetic algorithm based decision support system for the multi-objective node placement problem in next wireless generation network. Applied Soft Computing 33, 278-291. 
Abo-Zahhad, M., Ahmed, S.M., Sabor, N., Sasaki, S., 2015a. Rearrangement of mobile wireless sensor nodes for coverage maximization based on immune node deployment algorithm. Computers \& Electrical Engineering 43, 76-89.

Abo-Zahhad, M., Ahmed, S.M., Sabor, N., Sasaki, S., 2015b. Utilisation of multi-objective immune deployment algorithm for coverage area maximisation with limit mobility in wireless sensors networks. IET Wireless Sensor Systems 5, 250-261.

Cena, G., Seno, L., Valenzano, A., Zunino, C., 2010. On the performance of ieee 802.11e wireless infrastructures for soft-real-time industrial applications. Industrial Informatics, IEEE Transactions on 6, 425-437.

Chen, C.P., Mukhopadhyay, S.C., Chuang, C.L., Lin, T.S., Liao, M.S., Wang, Y.C., Jiang, J.A., 2015. A hybrid memetic framework for coverage optimization in wireless sensor networks. IEEE Transactions on Cybernetics 45, 2309-2322.

Cheng, Y.-S., Chuang, M.-T., Liu, Y.-H., Wang, S.-C., Yang, Z.-Z., 2016. A particle swarm optimization based power dispatch algorithm with roulette wheel redistribution mechanism for equality constraint. Renewable Energy 88, 58-72.

Gong, X., De Pessemier, T., Joseph, W., Martens, L., 2016a. A generic method for energy-efficient and energy-cost-effective production at the unit process level. Journal of Cleaner Production 113, 508-522.

Gong, X., Trogh, J., Braet, Q., Tanghe, E., Singh, P., Plets, D., Hoebeke, J., Deschrijver, D., Dhaene, T., Martens, L., Joseph, W., 2016b. Measurement-based wireless network planning, monitoring, and reconfiguration solution for robust radio communications in indoor factories. IET Science, Measurement \& Technology $10,375-382$.

Goudos, S.K., Plets, D., Liu, N., Martens, L., Joseph, W., 2015. A multi-objective approach to indoor wireless heterogeneous networks planning based on biogeography-based optimization. Computer Networks 91, 564-576.

Gupta, S.K., Kuila, P., Jana, P.K., 2016. Genetic algorithm approach for k-coverage and m-connected node placement in target based wireless sensor networks. Computers \& Electrical Engineering 56, 544-556.

Halder, S., Das Bit, S., 2015. Design of an archimedes' spiral based node deployment scheme targeting enhancement of network lifetime in wireless sensor networks. Journal of Network and Computer Applications 47, 147-167.

Hwu, W.-m., 2014. What is ahead for parallel computing. Journal of Parallel and Distributed Computing 74, 2574-2581.

Jaffres-Runser, K., Gorce, J.m., Ubeda, S., 2006. Multiobjective qos-oriented planning for indoor wireless lans, IEEE Vehicular Technology Conference, pp. 1-5.

Jain, A., Ramana Reddy, B.V., 2015. Eigenvector centrality based cluster size control in randomly deployed wireless sensor networks. Expert Systems with Applications 42, 2657-2669.

Jedda, A., Mouftah, H.T., 2016. Decentralized rfid coverage algorithms with applications for the reader collisions avoidance problem. IEEE Transactions on Emerging Topics in Computing 4, 502-515.

Johan, A., Mikael, G., Tomas, L., Krister, L., Mats, B., 2013. Design challenges and objectives in industrial wireless sensor networks, Industrial wireless sensor networks. CRC Press.

Ke, W.-C., Liu, B.-H., Tsai, M.-J., 2011. The critical-square-grid coverage problem in wireless sensor networks is np-complete. Computer Networks 55, 22092220.

Ke, W.C., Liu, B.H., Tsai, M.J., 2007. Constructing a wireless sensor network to fully cover critical grids by deploying minimum sensors on grid points is npcomplete. IEEE Transactions on Computers 56, 710-715.

Liao, W.-H., Kao, Y., Li, Y.-S., 2011a. A sensor deployment approach using glowworm swarm optimization algorithm in wireless sensor networks. Expert Systems with Applications 38, 12180-12188.

Liao, W.-H., Kao, Y., Wu, R.-T., 2011b. Ant colony optimization based sensor deployment protocol for wireless sensor networks. Expert Systems with Applications 38, 6599-6605.

Lin, Y.D., Tsao, S.L., Chang, S.L., Cheng, S.Y., Ku, C.Y., 2010. Design issues and experimental studies of wireless lan mesh. IEEE Wireless Communications $17,32-40$.

Liu, N., Plets, D., Goudos, S.K., Martens, L., Joseph, W., 2015a. Multi-objective network planning optimization algorithm: Human exposure, power consumption, cost, and capacity. Wireless Networks 21, 841-857.

Liu, N., Plets, D., Vanhecke, K., Martens, L., Joseph, W., 2015b. Wireless indoor network planning for advanced exposure and installation cost minimization. EURASIP Journal on Wireless Communications and Networking 2015, 1-14.

Liu, X., 2015. A deployment strategy for multiple types of requirements in wireless sensor networks. IEEE Transactions on Cybernetics 45, $2364-2376$.

Mukherjee, K., Gupta, S., Ray, A., Wettergren, T.A., 2011. Statistical-mechanics-inspired optimization of sensor field configuration for detection of mobile targets. IEEE Transactions on Systems, Man, and Cybernetics, Part B (Cybernetics) 41, 783-791.

Omidvar, M.N., Li, X., Tang, K., 2015. Designing benchmark problems for large-scale continuous optimization. Information Sciences 316, 419-436.

Park, J.H., 2016. All-terminal reliability analysis of wireless networks of redundant radio modules. IEEE Internet of Things Journal 3, $219-230$.

Plets, D., Tanghe, E., Paepens, A., Martens, L., Joseph, W., 2016. Wifi network planning and intra-network interference issues in large industrial warehouses, 2016 10th European Conference on Antennas and Propagation (EuCAP), pp. 1-5.

Rebai, M., Le berre, M., Snoussi, H., Hnaien, F., Khoukhi, L., 2015. Sensor deployment optimization methods to achieve both coverage and connectivity in wireless sensor networks. Computers \& Operations Research 59, 11-21.

Rizzi, M., Rinaldi, S., Ferrari, P., Flammini, A., Fontanelli, D., Macii, D., 2016. Enhancing accuracy and robustness of frequency transfer using synchronous ethernet and multiple network paths. IEEE Transactions on Instrumentation and Measurement 65, 1926-1936.

Senel, F., Akkaya, K., Erol-Kantarci, M., Yilmaz, T., 2015. Self-deployment of mobile underwater acoustic sensor networks for maximized coverage and guaranteed connectivity. Ad Hoc Networks 34, 170-183.

Siomina, I., Värbrand, P., Yuan, D., 2007. Pilot power optimization and coverage control in wcdma mobile networks. Omega 35, 683-696.

Sun, X., Zhang, Y., Ren, X., Chen, K., 2015. Optimization deployment of wireless sensor networks based on culture-ant colony algorithm. Applied Mathematics and Computation 250, 58-70.

Tang, L., Cao, H., Zheng, L., Huang, N., 2015. Rfid network planning for wireless manufacturing considering the detection uncertainty. IFAC-PapersOnLine 48, 406-411.

Tanghe, E., Gaillot, D.P., Liénard, M., Martens, L., Joseph, W., 2014. Experimental analysis of dense multipath components in an industrial environment. IEEE Transactions on Antennas and Propagation 62, 3797-3805.

Tanghe, E., Joseph, W., Verloock, L., Martens, L., Capoen, H., Herwegen, K.V., Vantomme, W., 2008. The industrial indoor channel: Large-scale and temporal fading at 900, 2400, and $5200 \mathrm{mhz}$. IEEE Transactions on Wireless Communications 7, 2740-2751.

Tian, J., Liang, X., Wang, G., 2016. Deployment and reallocation in mobile survivability-heterogeneous wireless sensor networks for barrier coverage. Ad Hoc Networks 36, Part 1, 321-331.

Vecchio, M., López-Valcarce, R., 2015. Improving area coverage of wireless sensor networks via controllable mobile nodes: A greedy approach. Journal of Network and Computer Applications 48, 1-13.

Wang, Y.M., Yin, H.L., Wang, J., 2009. Genetic algorithm with new encoding scheme for job shop scheduling. The International Journal of Advanced Manufacturing Technology 44, 977-984.

Yoon, Y., Kim, Y.H., 2013. An efficient genetic algorithm for maximum coverage deployment in wireless sensor networks. IEEE Transactions on Cybernetics 43, 1473-1483.

Zhang, T., Liu, J., 2017. An efficient and fast kinematics-based algorithm for rfid network planning. Computer Networks 121, 13-24. 
